Article

\title{
Studying State Convergence of Input-to-State Stable Systems with Applications to Power System Analysis
}

\author{
Antonio T. Alexandridis (D) \\ Department of Electrical and Computer Engineering, University of Patras, 26504 Patras, Greece; \\ a.t.alexandridis@ece.upatras.gr; Tel.: +30-261-099-6404
}

Received: 26 November 2019; Accepted: 19 December 2019; Published: 23 December 2019

\begin{abstract}
In stability studies, the response of a system enforced by external, known or unknown, inputs is of great importance. Although such an analysis is quite easy for linear systems, it becomes a cumbersome task when nonlinearities exist in the system model. Nevertheless, most of the real-world systems are externally enforced nonlinear systems with nonzero equilibriums. Representative examples in this category include power systems, where studies on stability and convergence to equilibrium constitute crucial objectives. Driven by this need, the aim of the present work is twofold: First, to substantially complete the theoretical infrastructure by establishing globally valid sufficient conditions for externally enforced nonlinear systems that converge to nonzero equilibriums and, second, to deploy an efficient method easily applicable on practical problems as it is analyzed in detail on a typical power system example. To that end, in the theoretical first part of the paper, a rigorous nonlinear analysis is developed. Particularly, starting from the well-established nonlinear systems theory based on Lyapunov techniques and on the input-to-state stability (ISS) notion, it is proven after a systematic and lengthy analysis that ISS can also guarantee convergence to nonzero equilibrium. Two theorems and two corollaries are established to provide the sufficient conditions. As shown in the paper, the main stability and convergence objectives for externally enforced systems are fulfilled if simple exponential or asymptotic converging conditions can be proven for the unforced system. Then, global or local convergence is established, respectively, while for the latter case, a novel method based on a distance-like measure for determining the region of attraction (RoA) is proposed. The theoretical results are examined on classic power system generation nonlinear models. The power system examples are suitably selected in order to effectively demonstrate the proposed method as a stability analysis tool and to validate all the particular steps, especially that of evaluating the RoA. The examined system results clearly verify the theoretical part, indicating a rather wide range of applications in power systems.
\end{abstract}

Keywords: stability; nonlinear systems; power system analysis; convergence to equilibrium

\section{Introduction}

Dynamic analysis, stability, and convergence to equilibrium constitute challenging issues in systems theory and applications and play a key role in evaluating system reliability and response. In practice, most of the systems are nonlinear, and this fact creates additional difficulties on conducting a systematic analysis on these issues. Indeed, most of the large complex systems, such as power systems, as well as smaller electromechanical apparatus in industry, belong to the nonlinear category. For their analysis, it has been clear that advanced methods and novel techniques are needed to be carefully studied in order to classify the most effective and crucial tools. Methods based on small signal analysis [1,2], or advanced Lyapunov techniques [3-5], have been extensively used. The former methods are capable to examine the dynamic system performance and stability, but as it is well-known, only local features can be analyzed whereas the validity of the whole analysis is straightforward 
dependent from the operation point and the system parameters. Furthermore, the small signal model has to be exclusively constructed from the particular original nonlinear system model, and none of the common characteristics (such as the natural energy functions) can be exploited in the analysis. Certainly, the main advantage of the method is that it permits the simple and well-known linear system techniques on both the time and frequency domain to be applied. In contrary, Lyapunov techniques are deployed by manipulating general storage functions that usually represent the energy handled by the particular system. Therefore, problems concerning the operation point or the system parameters are eliminated, and the analysis exploits the structural characteristics of the original nonlinear model of the system [6]. Besides the difficulties of constructing suitable Lyapunov functions, a fact relaxed in many practical systems where the natural energy functions can be used, a main drawback arises when external inputs enforce the system to nonzero equilibria that may vary in accordance to the input changes. Indeed, as the Lyapunov-based techniques are very efficient for systems having their equilibrium at the origin [7], the incremental nonlinear model or the linearized model via the Jacobian is often needed in the case of nonzero equilibriums. But because the incremental model in nonlinear cases has a clearly different form than the original one, the Lyapunov-based analysis becomes very difficult. On the other hand, linearization reintroduces the previously discussed certain drawbacks.

It is worth noting that among many different applications in nonlinear systems, power system analysis and control constitute very characteristic examples where all the aforementioned techniques have been extensively used. In their modern version, power systems are much more complex. They are externally enforced with a lot of locally controlled and uncontrolled inputs that act continuously in a wide range of varying equilibria due to the unpredictable nature of the renewable energy resources (RES) or the load demands, etc. [8]. Hence, new advanced methods of analysis are required that take into account all of these situations. In this field of advanced nonlinear system analysis and design, the notion of passivity was proposed for systems with external (known or unknown) inputs, which, however, does not directly contribute to stability issues of the forced system [9]. The notion of input-to-state stability (ISS), recently introduced by E. Sontag in 1989 in his famous paper [10], seems to fill this gap in nonlinear system theory. The kind of stability introduced by ISS provides a rigorous theoretical infrastructure capable of guaranteeing state boundedness for externally enforced nonlinear systems. The method is actually a Lyapunov-based one, since it has been proven that the existence of suitable Lyapunov functions is necessary and sufficient for the validity of the ISS property. It is noticed that besides from the globally valid ISS notion, systems can be examined for its local-ISS (l-ISS) property [11,12], whereas another significant feature, caused by the ISS notion, is that it implies the basic stability feature of externally driven systems, namely the bounded-input bounded-state (BIBS) property. Nevertheless, in most real-world systems, beyond stability (in the sense of BIBS) it is especially important to examine state convergence to a nonzero equilibrium for systems triggered by external bounded inputs, which are permanently acting and may converge to known or unknown nonzero constants [13].

Unfortunately, it has been proven [14] that ISS can additionally ensure convergence only to the zero equilibrium under the strict assumption that the bounded external input converges to zero. In this frame, this paper aims to bridge this gap with main objectives to complete the theoretical infrastructure by proving the sufficient conditions, which guarantee convergence to nonzero equilibriums for ISS (or l-ISS) systems when bounded external inputs converge to nonzero values and, to establish an effective tool for practical applications. Particularly, starting from the most difficult case of an $l$-ISS generally nonlinear system and considering an external input that converges to a constant, it is proven after lengthy manipulations that a suitable $C^{1}$ class, lower-bounded, storage function always exists, which satisfies all the conditions of the local Invariant Principle (LaSalle Theorem). As proven in the paper, the time derivative of this function is negative semidefinite, i.e., it is actually negative definite outside and zero inside of a compact set that involves the set of all the system equilibriums. Thus, the system trajectories tend to this set, wherein they stay for all time thereafter and, in accordance to the LaSalle Invariant Principle, the trajectories eventually converge to equilibrium. A simple extension of 
the above procedure provides the global version for convergence of the states in the case of purely ISS systems. Hence, making use of the definitions and preliminaries described in Section 2, two theorems are established in Section 3: One for local and the other for global convergence of a $l$-ISS and ISS system, respectively. Further relaxed conditions are established in Corollaries 1 and 2 for the two cases discussed, which pass beyond the need to intermediately prove the $l$-ISS or ISS property. Therefore, using the Corollaries, it is sufficient to examine the unforced system for its asymptotic or exponential stability for each case, respectively. As a result, traditional and well-known in the literature methods for the unforced system are adequate for proving convergence to the equilibrium of the externally enforced system. However, in Section 4, the significant issue of evaluating the region of attraction (RoA) in the case of local convergence is confronted by introducing a suitable metric (distance-like measure) and a systematic procedure of analysis.

The theoretical results are applied in Section 5 on the analysis of classic power system dynamic studies. Electromechanical dynamic effects in power systems occurred by small or large disturbances are very important for the system stability [1]. As single- or multistage- turbine driven generators constitute the usual case, it is very convenient to exhibit the effectiveness of the proposed method in such power system configurations, since there are represented by relatively low-order nonlinear models where one can easily understand the implementation of the proposed procedure. The focus is especially given to stability studies and on the evaluation of the RoA. In large-scale systems, it is not always an easy task to directly obtain the l-ISS or ISS property. However, the situation is substantially simplified when the problem is transferred on what is known for the asymptotic or exponential stability (AS or ES) characteristics of the unforced system around zero. In the first case of AS, a careful procedure is additionally needed to determine the permitted regions inside which the states and the external inputs should be lying. In any case, the procedure needs only the state space representation of the whole system, accompanied by some common conditions, as preliminaries. As proven in the paper, the method is clearly accurate since it is applicable on the nonlinear system model, it is easily applied since it can exploit the a priori known natural energy expressions as suitable Lyapunov-type storage functions and it can provide a very satisfactory solution for the RoA determination. Simulation studies that involve a gradual evolution of the power plant and control system into more complex schemes fully verify the validity of the proposed analysis approach and indicate its effectiveness in real-world power system problems. Consequently, it is apparent that this method can provide a fundamental and general tool for the analysis and design of more complex power systems, a fact that is expected to be exploited in the near future.

\section{Notations, Definitions and Preliminaries}

In this paper, finite-dimensional nonlinear systems of the form of (1), enforced by external inputs, are considered:

$$
\dot{x}(t)=f(x(t), u(t))
$$

where state $x(t) \in D$, input $u(t) \in D_{u}$, and $f: D \times D_{u} \rightarrow \mathbb{R}^{n}$ is piecewise continuous in $t$ and at least locally Lipschitz in $x$ and $u$. The sets $D$ and $D_{u}$ are defined as follows:

$$
D=\left\{x \in \mathbb{R}^{n}:\|x\| \leq r\right\}, D_{u}=\left\{u \in \mathbb{R}^{m}: \sup _{t \geq 0}\|u\|=\|u\|_{L \infty} \leq r_{u}\right\}
$$

It is assumed that $f(0,0)=0$. Following the notations and definitions given in [11,15], we denote the Euclidean norm as $\|x\|$ in this paper. For a function of time, usually an input, the (essential) supremum norm is denoted by (ess) $\sup _{t \geq 0}\|u\|=\|u\|_{L_{\infty}}$. That is, $\|u\|_{L_{\infty}}$ is the smallest number $k$ such that $\|u(t)\| \leq k$ for (almost) all $t \geq 0$.

A continuous function $a(s)$ is a class $\mathcal{K}$ function [16], if it is strictly increasing and $a(0)=0$. It is of class $\mathcal{K}_{\infty}$ if additionally $a(s) \rightarrow \infty$ as $s \rightarrow \infty$. A continuous function $\beta(s, p)$ is a class $\mathcal{K} \mathcal{L}$ function if for each fixed $p$, it belongs to class $\mathcal{K}$ with respect to $s$, and for each fixed $s$, it is decreasing with respect to $p$ and $\beta(s, p) \rightarrow 0$ as $p \rightarrow \infty$. Denoting the inverse of $a(s)$ by $a^{-1}(s)$, then this belongs to class $\mathcal{K}$ or 
to $\mathcal{K}_{\infty}$ if $a(s)$ belongs to class $\mathcal{K}$ or to $\mathcal{K}_{\infty}$, respectively. For two (or more) class $\mathcal{K}$ (or $\mathcal{K}_{\infty}$ ) functions, the composite $a_{1} \circ a_{2}(s)$ denotes the $a_{1}\left(a_{2}(s)\right)$ and belongs to class $\mathcal{K}$ (or $\left.\mathcal{K}_{\infty}\right)$ functions if at least one of them is class $\mathcal{K}$ (or all of them are class $\mathcal{K}_{\infty}$ ).

Before proceeding with the basic preliminaries for ISS and convergence issues, a certain terminology, commonly used for the system characterization, should be clarified [9].

Definition 1. A system of the general form $\dot{x}(t)=f(x(t), t)$ is characterized as non-autonomous system whereas a system $\dot{x}(t)=f(x(t))$ is characterized as autonomous system.

An example of a non-autonomous system is $\dot{x}(t)=f(x(t), u(t))$ for any $u(t)$ to be a function of time. Clearly, the unforced system $\dot{x}(t)=f(x(t), 0)$ and, the system $\dot{x}=f(x(t), \bar{u})$ for some $\bar{u}$ constant are both autonomous systems.

Definition 2. [9,11]. The system (1) is defined to be locally input-to-state-stable (l-ISS) if there exists a class $\mathcal{K} \mathcal{L}$ function $\beta$ and a class $\mathcal{K}$ function $\gamma$ and positive constants $k_{1}$ and $k_{2}$ such that

$$
\|x(t)\| \leq \beta(\|x(0)\|, t)+\gamma\left(\sup _{0 \leq \tau \leq t}\|u(\tau)\|\right)
$$

for all $t \geq 0$ and $x \in D, u \in D_{u}$ with $\|x(0)\|<k_{1}$ and, (ess) $\sup _{t \geq 0}\|u\|=\|u\|_{L_{\infty}}<k_{2}$. The system is said to be input-to-state-stable (ISS) if $D=\mathbb{R}^{n}, D_{u}=\mathbb{R}^{m}$ and (3) holds true for any initial state $x(0)=x_{0}$ and any bounded input $u(t)$.

The following Theorem provides the necessary and sufficient conditions for suitable Lyapunov-type storage functions $[11,17]$ to admit $l$-ISS (or ISS) in accordance to Definition 2.

Theorem 1. System $\dot{x}=f(x, u)$ with $f$ as defined in (1) is l-ISS if and only if there exists a continuously differentiable function $V: D \rightarrow \mathbb{R}$ with $a_{1}, a_{2}, a_{3}$ and $\sigma$ class $\mathcal{K}$ functions such that

$$
\begin{gathered}
a_{1}(\|x\|) \leq V(x) \leq a_{2}(\|x\|) \\
\frac{\partial V}{\partial x} f(x, u) \leq-a_{3}(\|x\|)+\sigma(\|u\|)
\end{gathered}
$$

for all $t \geq 0$ and $x \in D, u \in D_{u}$. Then function $V$ is called an ISS Lyapunov function on $D$. If $D=\mathbb{R}^{n}$, $D_{u}=\mathbb{R}^{m}$ and $a_{1}, a_{2}, a_{3}$ and $\sigma$ are class $\mathcal{K}_{\infty}$ functions then the system (1) is ISS and, function $V$ is called an ISS Lyapunov function.

Alternatively, Theorem 1 holds true if Inequality (5) is replaced by:

$$
\frac{\partial V}{\partial x} f(x, u) \leq-a_{4}(\|x\|) \forall\|x\| \geq \rho(\|u\|)
$$

with $a_{4}, \rho$ class $\mathcal{K}$ functions for $l$-ISS and class $\mathcal{K}_{\infty}$ functions for ISS. Where needed, parameter $\gamma$ is calculated by $\gamma=\alpha_{1}^{-1} \circ \alpha_{2} \circ \rho$, while bounds $k_{1}$ and $k_{2}$ are defined as: ·

$$
\begin{gathered}
k_{1}=\alpha_{2}^{-1} \circ \alpha_{1}(r) \\
k_{2}=\rho^{-1}\left(\min \left\{k_{1}, \rho\left(r_{u}\right)\right\}\right)
\end{gathered}
$$

From [11,12], the following Lemma is recalled, which suggests that asymptotic stability (AS) of the unforced system implies $l$-ISS. For rigorous analysis of this result, one can see the first part of the proof of Theorem 5.2 in ([9] 3rd Ed.). It is noticed that in earlier publications, it has been proposed and proven [18] that global-AS implies l-ISS, but the Lemma that follows $[9,11]$ substantially relaxes this necessity. 
Lemma 1. If the origin of the unforced autonomous system $\dot{x}=f(x, 0)$ is asymptotically stable and the function $f(x, u)$ is continuously differentiable, then system (1) is l-ISS.

In the next Lemma [9], the ISS property is related to the exponential stability of the unforced system, but now the requirement of globally Lipschitz property replaces the initial assumption of the locally Lipschitz property of $f$ in $x$ and $u$, a fact that is difficult to be satisfied.

Lemma 2. Suppose that $f(x, u)$ is continuously differentiable and globally Lipschitz in $x$ and $u$. If the origin of the unforced autonomous system $\dot{x}=f(x, 0)$ is exponentially stable, then system (1) is ISS.

Another interesting result is presented via Theorem 2 [19], which makes possible to study the convergence of a system of the form of (1) to a nonzero equilibrium as $t \rightarrow \infty$.

Theorem 2. Suppose that for the nonautonomous system $\dot{x}(t)=f(x(t), u(t))$, the input $u(t) \rightarrow \bar{u}$ as $t \rightarrow \infty$, for some $\bar{u}$ constant. Then, $x(t) \rightarrow x^{*}$ as $t \rightarrow \infty$ if this $x^{*}$ is an asymptotically stable (AS) equilibrium of the autonomous system

$$
\dot{x}=f(x, \bar{u})
$$

in a domain of attraction $D_{A}$ with $x(\cdot)$ to be a K-recurrent solution of the original system (1) defined on $[0, \infty)$, in a set $K$ which is a compact subset of $D_{A}$.

In Theorem 2, the terminology used for K-recurrency is defined as follows [19]:

Definition 3. Given a compact subset $K \subseteq X$, we say that a function $x(t) \in X$ is $K$-recurrent if for each $T>0$ there is some $t>T$ such that $x(t) \in K$.

According to Theorem 2, if one can prove that under the K-recurrency property, the (autonomous) system enforced by a permanently constant input is AS around a nonzero equilibrium, then the nonautonomous system enforced by a bounded, continuous, input function that tends to the aforementioned constant also converges to the same nonzero equilibrium. Following this concept, the basic problem of proving convergence to the equilibrium for systems that are enforced by a $u(t) \rightarrow \bar{u}$ as $t \rightarrow \infty$ and satisfy the l-ISS or ISS property is still open with an apparent benefit that it is now modified to an autonomous system stability problem with $u(t) \equiv \bar{u}$. Hence, the powerful invariant set theorems for autonomous systems, known as LaSalle invariant principles, can be used in the theoretical analysis. These principles are recalled from $[7,9]$ and are presented in the following.

Theorem 3. (Local Invariant Set Theorem). Consider an autonomous system, for instance, of the form (9), with $f$ continuous, and let $W(x)$ be a scalar function with continuous first partial derivatives ( $C^{1}$ class). Assume that for some $l>0$, the region $S_{l}$ defined by $W(x)<l$ is bounded and $\dot{W}(x) \leq 0$ for all $x$ in $S_{l}$. Let $\Omega$ be the set of all points within $S_{l}$ where $\dot{W}(x)=0$ and $E$ be the largest invariant set in $\Omega$. Then every solution $x(t)$ originating in $S_{l}$ asymptotically tends to $E$ as $t \rightarrow \infty$.

Theorem 4. (Global Invariant Set Theorem). Consider an autonomous system, for instance, of the form (9), with $f$ continuous, and let $W(x)$ be a scalar function with continuous first partial derivatives ( $C^{1}$ class). Assume that $\dot{W}(x) \leq 0$ over the whole state space, i.e., for all $x \in \mathbb{R}^{n}$ with $W(x) \rightarrow \infty$ as $\|x\| \rightarrow \infty$. Let $\Omega$ be the set of all points where $\dot{W}(x)=0$ and $\mathrm{E}$ be the largest invariant set in $\Omega$. Then all solutions globally asymptotically converge to $E$ as $t \rightarrow \infty$.

In Theorems 3 and 4 , if the set $\Omega$ defined by $\dot{W}(x)=0$ contains only a unique equilibrium, then the state trajectories asymptotically converge to that point.

Another significant point met in the theoretical part of analysis is related with the answer on the critical question: How one can determine the region of attraction (RoA) in the cases when local 
convergence to equilibrium has been proven. Many attempts exist in the literature, which provide a more or less satisfying answer on this issue [20]. However, in this case, the problem of determining the RoA is confronted by introducing a novel metric around the equilibrium, based on the notion of Bregman distance (or divergence) [21], which is defined as follows.

Definition 4. Let function $h$ be strictly convex in a closed convex set $\Omega_{h} \subseteq \mathbb{R}^{n}$ such that $h$ is continuously differentiable on relative interior of $\Omega_{h}$. Then, the distance measure (Bregman distance) $\Omega_{h} \times \Omega_{h} \rightarrow \mathbb{R}$ is defined as

$$
M_{p q}(p, q)=h(p)-h(q)-\langle\nabla h(q), p-q\rangle
$$

where $\langle\cdot, \cdot\rangle$ denotes inner product operator.

The Bregman distance has been already used in many applications, from data mining and machine learning [22] to control innovations [23]. Nevertheless, the distance measure $M_{p q}$ is not necessarily a true distance in the sense of metric topology and it can be characterized as a distance-like metric for convex functions. Since $\forall(p, q) \in \Omega_{h} \times \Omega_{h}$, it is:

$$
M_{p q}(p, q) \geq 0
$$

due to the convexity property: $h(p) \geq h(q)+\langle\nabla h(q), p-q\rangle$ of the function $h(p)$ around point $q$, and

$$
M_{p q}(p, q)=0 \Leftrightarrow p=q
$$

but may not satisfy other distance properties, such as the triangle inequality and symmetry (i.e., in general it holds true: $\left.M_{p q}(p, q) \neq M_{q p}(q, p)\right)$.

It is also clear that $M_{p q}$ may be interpreted as the distance measure of the $h(p)$ function from its value $h(q)$ around a fixed point $q$, for any $p$ ensuring convexity of $h$.

Now, following the notations and definitions presented, our theoretical results on the basis of the ISS properties and Lyapunov-based techniques can be established.

\section{Main Results on Converging Properties of ISS Systems}

To proceed with our main results, we start with some remarkable comments on the fundamental notion of ISS as they are pointed out in the literature.

The rigorous definition of ISS and the whole theory developed around this notion [24] mainly aims to cover issues in nonlinear system analysis, as these are related to a system response when permanent external inputs take the state trajectories away from the zero equilibrium at the origin. Hence, ISS clearly examines the stability properties of an externally enforced system in contrary to dissipative and passivity properties that simply provide input-output relations [9]. Specifically, in [25] it is referred: ISS states the fact that if the inputs are uniformly small then the system states must be eventually small; an alternative statement of the BIBS property. On the other hand, convergence to equilibrium for ISS systems is proven only for the zero equilibrium when the external input tends to zero [17]. The above descriptions indicate that stability aspects for ISS systems are still seen under the zero-equilibrium point of view. In this paper, however, we concentrate on stability aspects and sufficient conditions for state convergence of ISS systems to nonzero equilibria imposed by permanently nonzero external inputs. Two different cases are considered: One is related to l-ISS systems, while the other simply extends the results for the pure ISS systems case.

\subsection{A Pass from ISS Lyapunov Functions to Suitable Storage Functions for the LaSalle Invariant Principles}

As the title of this subsection briefly describes, convergence to nonzero equilibrium for l-ISS or ISS systems will be proven via the appropriate application of the LaSalle Invariant Principles. To do this task, it is necessary to prove that l-ISS and/or ISS lead to the construction of suitable storage functions. 
We first assume that system (1) is l-ISS. In this case, Theorem 1 holds true and a well-known $[9,11]$, first-pass from inequality (5) to that of (6) can be implemented with $a_{4}(\|x\|)=(1-\theta) a_{3}(\|x\|)$ and $\rho(\|u\|)=a_{3}^{-1}\left(\frac{\sigma\|u\|}{\theta}\right)$ for any $\theta: 0<\theta<1$. Therefore, by considering a predefined $r_{u}$ and $r$ large enough, it is concluded from (7) and (8) that the maximum of $k_{1}, k_{2}$ occur for $k_{2}=r_{u} \equiv($ ess $)$ sup $\|u\|_{L \infty}$, and, consequently, for $k_{1}=\rho\left(r_{u}\right)$, which is now a function of $\theta$. This $\theta$ parametrization actually provides a degree of freedom on determining different bounds on $\|x\|$, which however cannot be further exploited in the analysis. It is also noticed that one can consider the $k_{1, \max }=\rho\left(r_{u}\right)_{\max }$ where $\rho\left(r_{u}\right)_{\max } \rightarrow a_{3}^{-1}\left(\frac{\sigma\left(r_{u}\right)}{\theta}\right)$ as $\theta \rightarrow 1$.

Nevertheless, an alternative and more constructive pass from (5) to (6) is realized in the present analysis by splitting the first right-hand side term of inequality (5) into:

$$
\begin{gathered}
\left.-a_{3}(\|x\|)=-a_{3}(\|x\|)+a_{3} \circ a_{2}^{-1} \circ a_{1}(\|x\|)-a_{3} \circ a_{2}^{-1} \circ a_{1}(\|x\|)\right)= \\
\left.-a_{3} \circ a_{2}^{-1} \circ\left(a_{2}-a_{1}\right)(\|x\|)-a_{3} \circ a_{2}^{-1} \circ a_{1}(\|x\|)\right)
\end{gathered}
$$

It is noticed that (4) implies

$$
a_{2}(s) \geq a_{1}(s)
$$

and, without loss of generality, even if $a_{2}(s)=a_{1}(s)$, one can assume some slightly different functions in (4) which satisfy the pure inequality $a_{2}(s)>a_{1}(s)$. As a direct result, condition (6) is concluded from (5) if (13) is taken into account, with

$$
\dot{V} \leq-a_{3} \circ a_{2}^{-1} \circ\left[a_{2}-a_{1}\right](\|x\|) \forall\|x\| \geq \bar{\rho}(\|u\|)
$$

with $\bar{\rho}(\|u\|)=\bar{a}^{-1} \circ \sigma(\|u\|)$, where $\bar{a}(s)=a_{3} \circ a_{2}^{-1} \circ a_{1}(s), \bar{a}(s) \in \mathcal{K}$ and $a_{3} \circ a_{2}^{-1} \circ\left[a_{2}-a_{1}\right](\|x\|) \in \mathcal{K}$.

But the most significant result of this formulation comes from the fact that since (13) and (14) hold true, it is $a_{3}(s) \geq \bar{a}(s)$ and then $\dot{V}$ as given by (5) can be transferred into:

$$
\dot{V} \leq-\bar{a}(\|x\|)+\sigma(\|u\|)
$$

Therefore, $k_{1}$ is determined by

$$
k_{1}=\bar{\rho}\left(r_{u}\right)
$$

and

$$
r=a_{1}^{-1} \circ a_{2}\left(k_{1}\right)=a_{1}^{-1} \circ a_{2} \circ \bar{\rho}\left(r_{u}\right)
$$

Note that $k_{1}<r$, since $a_{1}(s)<a_{2}(s)$ is assumed.

Also, as it is easily obtained by the initial formulation of the $\dot{V}$ in (5), this can also be in the form [17],

$$
\dot{V} \leq-a(V)+\sigma(\|u\|) \text { with } a(s)=a_{3} \circ a_{2}^{-1}(s) \in \mathcal{K}
$$

Now, defining the compact set (closed and bounded set):

$$
S=\{x \in D: V(x) \leq c\} \text { with } c \equiv a_{2}(r)>a_{2}\left(k_{1}\right)
$$

then, it holds sequentially

$$
\|x\|<k_{1}<r \Rightarrow a_{2}(\|x\|)<a_{2}\left(k_{1}\right)<c
$$

and since we have by the right-hand side of (4), $V \leq a_{2}(\|x\|)$, it is implied that the open set

$$
S_{o}=\left\{x \in \mathbb{R}^{n}:\|x\|<k_{1}\right\} \subset S \subseteq D
$$

Thus, for any $\left\|x_{0}\right\|<k_{1}, l$-ISS holds true for all $x \in D$ and $u \in D_{u}$. 
Now, consider that the external (bounded) input $u(t)$ is such that $u(t) \rightarrow \bar{u}$ as $t \rightarrow \infty$ with $\|u\|_{L_{\infty}} \in D_{u}$. Our purpose is to prove that also $x \rightarrow x *$ as $t \rightarrow \infty$, with $x * \in D: f(x *, \bar{u})=0$ to denote the equilibria as $t \rightarrow \infty$.

To proceed with our task, we recalled Theorem 2. In accordance to Theorem 2, it is now sufficient to prove that the system $\dot{x}=f(x, \bar{u})$ converges to some equilibrium $x * \in E$, where $E=$ $\left\{x * \in \mathbb{R}^{n}: f(x * \bar{u})=0\right\}$ is the largest invariant set of $\dot{x}=f(x, \bar{u})$.

It is worth noting that l-ISS property satisfies the assumptions of Theorem 2, i.e., the K-recurrency property. Furthermore, since the latter system for $u=\bar{u}=$ const has now become an autonomous system, it is sufficient to apply the LaSalle Invariance Principle (Theorem 3) in order to prove stability and convergence to $E$.

Certainly, to accomplish the analysis, a suitable storage function is needed. To that end, one can first observe that given $\|\bar{u}\|<r_{u}$ there exists a constant $\bar{u}_{d}:\|\bar{u}\|<\bar{u}_{d}<r_{u}$ and two different $\bar{x}_{1}, \bar{x}_{2} \in \mathbb{R}^{n}$ such that

$$
\bar{a}\left(\left\|\bar{x}_{1}\right\|\right)=\sigma\left(\left\|\bar{u}_{d}\right\|\right) \text { and } a\left(V\left(\bar{x}_{2}\right)\right)=\sigma\left(\left\|\bar{u}_{d}\right\|\right)
$$

Since from (21), $\left\|\bar{x}_{1}\right\|=\bar{a}^{-1}\left(\sigma\left(\left\|\bar{u}_{d}\right\|\right)\right)$ and by definition $k_{1}=\bar{\rho}\left(r_{u}\right) \equiv \bar{a}^{-1}\left(\sigma\left\|r_{u}\right\|\right)$, it is implied $\left\|\bar{x}_{1}\right\|<k_{1}$.

Denoting $\bar{c}=\sigma\left(\left\|\bar{u}_{d}\right\|\right)$ and defining the set

$$
\Omega=\left\{x \in \mathbb{R}^{n}: V(x) \leq a^{-1}(\bar{c})\right\}
$$

then, taking into account (21) and some other properties as previously discussed, the following can be sequentially obtained:

$$
a^{-1}(\bar{c})=V\left(\bar{x}_{2}\right)=a^{-1} \circ \bar{a}\left(\left\|\bar{x}_{1}\right\|\right)=a_{1}\left(\left\|\bar{x}_{1}\right\|\right)<a_{2}\left(\left\|\bar{x}_{1}\right\|\right)<a_{2}\left(\left\|k_{1}\right\|\right)<c
$$

Thus, from (23), it is clearly concluded that

$$
\Omega \subset S_{0} \subset S \subseteq D
$$

A second remark can be made by considering $\dot{V}$ for $u=\bar{u}$ as determined by (18):

$$
\dot{V} \leq-a(V(x))+\sigma(\|\bar{u}\|)<-a(V(x))+\bar{c} .
$$

Then, apparently $\dot{V}=\frac{\partial V}{\partial x} f(x, \bar{u})=0$ at the equilibrium $x=x * \in E$, which in accordance to the latter inequality it implies $a(V(x *))<\bar{c}$ or $V(x *)<a^{-1}(\bar{c})$, which in turn means that $E \subset \Omega$. Therefore, by considering (24), it is eventually proven

$$
E \subset \Omega \subset S_{o} \subset S \subseteq D
$$

Now, after the above remarks we propose for the system $\dot{x}=f(x, \bar{u}), \forall x \in D$ the following storage function

$$
W(x(t))=\left\{\begin{array}{clc}
\frac{1}{2}[a(V(x))-\bar{c}]^{2}, & \text { for } V(x)>a^{-1}(\bar{c}), & (x \in D / \Omega) \\
0, & \text { for } V(x) \leq a^{-1}(\bar{c}), & (x \in \Omega)
\end{array}\right.
$$

It is worth noting that the storage function $W(x)$ is lower bounded and $C^{1}$ class on $D$.

\subsection{Proving Convergence to Nonzero Equilibrium}

As shown in the previous subsection, storage function (26) can be considered as the required function for examining system $\dot{x}=f(x, \bar{u})$ in accordance to Theorem 3 (Local Invariant Set Theorem). 
Our aim is to prove that the $l$-ISS system states converge to their equilibriums in $E$. To that end, the time derivative of $W$ is calculated as follows:

$$
\begin{aligned}
& \dot{W}=\frac{\partial W}{\partial a} \frac{\partial a}{\partial t}=\left\{\begin{array}{c}
{[a(V(x))-\bar{c}](\dot{a}(V))} \\
0
\end{array}\right. \\
& =\left\{\begin{array}{cc}
{[a(V(x))-\bar{c}]\left(\frac{\partial a}{\partial V}\right) \dot{V}<-[a(V(x))-\bar{c}]^{2}\left(\frac{\partial a}{\partial V}\right)<0,} & \text { for } V(x)>a^{-1}(\bar{c}) \\
0, & \text { for } V(x) \leq a^{-1}(\bar{c})
\end{array}\right.
\end{aligned}
$$

From (27), it is observed that since $\dot{W}<0$ outside of $\Omega$, every solution $x(t)$ of $\dot{x}=f(x, \bar{u})$ in $D$, tends to $\Omega$, wherein it remains all time thereafter. Since (25) holds true, it is immediately implied that applying the LaSalle Invariance Principle in the region $V(x) \leq c$ it is proven that every trajectory $x(t)$ in $D$ of the autonomous system $\dot{x}=f(x, \bar{u})$ approaches $E$ as $t \rightarrow \infty$. Consequently, as Theorem 2 indicates, it is concluded that every trajectory $x(t)$ in $D$ of the $l$-ISS system $\dot{x}=f(x, u)$ approaches $E$ as $t \rightarrow \infty$. Furthermore, if $f(x *, \bar{u})=0$ determines a unique $x *$, then every solution $x(t)$ in $D$ converges asymptotically to $x *$.

Therefore, from the previous analysis, the following Theorem is established for the original $l$-ISS system (1).

Theorem 5. For system $\dot{x}(t)=f(x(t), u(t))$, l-ISS property implies that $\forall x(t) \in D, u(t) \in D_{u}$, the trajectories $x(t) \rightarrow x^{*} \in E$ for a $u(t) \rightarrow \bar{u}$ as $t \rightarrow \infty$, for some $\bar{u}$ constant.

Nevertheless, Theorem 5 can be further relaxed by considering Lemma 1 . Hence the following Corollary is also established.

Corollary 1. For system $\dot{x}(t)=f(x(t), u(t))$, if the origin of the unforced autonomous system $\dot{x}=f(x, 0)$ is asymptotically stable and the function $f(x, u)$ is continuously differentiable, then $\forall x(t) \in D, u(t) \in D_{u}$ the trajectories of the enforced system converge to equilibrium: $x(t) \rightarrow x^{*} \in E$ for a $u(t) \rightarrow \bar{u}$ as $t \rightarrow \infty$, for some $\bar{u}$ constant.

For the pure ISS systems case, it becomes apparent that the aforementioned analysis holds true $\forall x(t) \in \mathbb{R}^{n}$ and $\forall u(t) \in \mathbb{R}^{m}$ bounded. Hence, in a similar manner, the storage function (26) can be used $\forall x(t) \in \mathbb{R}^{n}, u(t) \in \mathbb{R}^{m}$ in Theorem 4 (Global Invariant Set Theorem), which, in combination with Theorem 2, establishes the following result:

Theorem 6. For system $\dot{x}(t)=f(x(t), u(t))$, ISS property implies that $\forall x(t) \in \mathbb{R}^{n}, u(t) \in \mathbb{R}^{m}$ the trajectories $x(t) \rightarrow x^{*} \in E$ for a $u(t) \rightarrow \bar{u}$ as $t \rightarrow \infty$, for some $\bar{u}$ constant.

Additionally, in this case, Theorem 6 can be further relaxed in the following Corollary by considering Lemma 2.

Corollary 2. For system $\dot{x}(t)=f(x(t), u(t))$, if the origin of the unforced autonomous system $\dot{x}=f(x, 0)$ is exponentially stable and the function $f(x, u)$ is continuously differentiable and globally Lipschitz in $x$ and $u$, then $\forall x(t) \in \mathbb{R}^{n}$ and $\forall u(t) \in \mathbb{R}^{m}$ bounded, the trajectories of the enforced system converge to equilibrium: $x(t) \rightarrow x^{*} \in E$ for a $u(t) \rightarrow \bar{u}$ as $t \rightarrow \infty$, for some $\bar{u}$ constant.

At this point, it is significant to make some comments and remarks as follows.

The procedure developed for the proofs of Theorems 5 and 6 indicates that $l$-ISS and ISS are the sufficient conditions for the existence of the suitable storage functions of the form of (26) for each case respectively. Clearly, in any case, it is not needed to construct (26) and to calculate its time derivative (27). Their existence adequately guarantees the convergence to nonzero equilibriums. Furthermore, as Corollaries 1 and 2 indicate, the whole stability and convergence analysis to nonzero equilibriums of 
externally enforced nonlinear systems was modified to that of examining the convergence to the origin of the unforced system. Apparently, the latter constitutes a standard and well-established problem in Lyapunov based nonlinear system theory (see, for example, [7,9] and the references therein). Moreover, in practical applications, such as in power system studies, the construction of suitable Lyapunov storage functions is implemented for the unforced system by simply considering the sum of the natural energy of the different system parts.

It is also noticed that relatively recent research works [26,27] have attempted to confront this problem for BIBS systems and for the special case of port Hamiltonian systems under some certain and strict conditions. In the present work, substantially generic results were proven with actually no need for additional constraints.

Finally, it is remarkable to note that the theoretical analysis as previously deployed was based on either the l-ISS property, which holds true in a permitted RoA, or on the pure ISS property, which is globally valid. As for practical applications, locally converging properties to nonzero equilibriums constitute the usual case, it is very important to determine the domain wherein these stability properties are valid.

\section{Evaluating the RoA around the Equilibrium}

In the cases where local convergence to equilibrium is proven, it is immediately raised the question of how one can determine the RoA around this equilibrium. It is obvious that if Theorem 5 is applied and conditions (4) and (5) can be analytically obtained, then the bounds given by (7) and (8) can be also easily obtained. However, when Corollary 1 is used, it is not easily possible for the conditions (4) and (5) to be analytically obtained. In that case, it is needed to follow alternative ways for determining the RoA, a fact very important for the system performance since AS of the unforced system is not adequate to maintain stability when $u(t) \neq 0$, even if $u(t)$ is very small [17]. Even when global AS has been proven for the unforced system, there are counterexamples where $x(t)$ does not converges to zero or diverges from the initial time instant, although $u(t)$ vanishes as $t$ tends to infinity if, initially, the states are lying outside from the permitted region [14]. It is also noticed that during the period when $u(t)$ converges to $\bar{u}$, its deviation from $\bar{u}$ should be small enough in order to keep $u(t)$ inside its permitted region, a fact that has to be carefully taken into account in any practical application. For example, this is satisfied for piecewise constant input changes when the initial value before every such a change to lie inside the permitted region. Additionally, for any selected constant $\bar{u}$, a system equilibrium must exist. Violation of this condition leads to completely unstable situations as characteristic counterexamples in previous literature has indicated, see e.g., the counterexample in [9] after Lemma 4.6 where the constant input $u=1$ results in divergences.

In accordance with Corollary 1, it is sufficient to prove AS around zero for the unforced system that according to the well-known Lyapunov techniques is based on a suitable storage function, let $H(x)$, with negative definite or negative semidefinite time derivative [9]. Hence, to easily implement the task of evaluating a nonconservative and large enough RoA for this case, the known $H(x)$ is proposed to be used for the RoA determination. It is, therefore, noticed that for any $x$ close to $x=0$, an estimate of $H(x): \hat{H}(x)=H(0)+\langle\nabla H(0), x-0\rangle$ can be defined for the unforced system and then a closed bounded region $H(x)<c_{L}\left(c_{L}>0\right)$ exists as long as the convexity property $H(x) \geq \hat{H}(x)$ holds true. Such a capable of guaranteeing convexity $x$ exists in the common case where $H$ used for proving stability around zero is positive definite (globally or locally). Extending this aspect for any particular nonzero equilibrium point $x *$ on $H(x)$, which may be not a pure convex function (such as, for example, quadratic functions are) and may involve a convex domain (perhaps close to zero as discussed for positive definite $H$ ) wherein $x *$ belongs, it is introduced a generic suitable metric, capable of sharing the maximum possible limits around the equilibrium where the stability constraints hold true. To this end, the distance-like measure as provided in Definition 4 is recalled and applied for $q=x *$ and $p$ 
being any $x$ around the equilibrium $x *$, i.e., considering $h=H(x)$ to be the relative Lyapunov function, then from (10) the following distance measure is proposed:

$$
M_{x x *}(x, x *)=H(x)-H(x *)-\langle\nabla H(x *), x-x *\rangle
$$

as long as $M_{x x *} \geq 0$.

Then, to construct a compact RoA in the convex domain around $x *$, it is necessary for $M_{x x *}$ to perform like a local storage function with $\dot{M}_{x * *} \leq 0$ [9,11], and for all the permitted $x$ to belong in a domain of $H(x)$ such that the lines passing through $x *$ and the arbitrary $x$ to ensure local convexity of $H(x)$, i.e., all the points of $H$ between $x *$ and $x$ to lie below of the line $x *-x$. This means that all permitted $x$ should satisfy:

$$
H(x)-H(x *) \leq\langle\nabla H(x), x-x *\rangle
$$

The maximum $x$ under these circumstances can then be defined by the maximum region around $x *$ where the above convexity condition of $H$ holds true, and it is actually defined as the unique intersection points of $H(x)$ with the lines passing through $x *$ and are tangent to the function $H(x)$. Among all these candidate points, the one closest to $x *$ is obviously selected. Thus, the $x_{\max }$ is obtained when inequality (29) reaches its upper limit, i.e., when $H(x *)-H(x)-\langle\nabla H(x), x *-x\rangle=0$.

From the latter expression, and after taking into account the previous remarks, one can easily see the interesting result that the maximum $x$ is obtained by constraining the symmetric of the distance measure (28), namely the $M_{x * x}(x *, x)$, to be equal to zero under the following conditions:

$$
x_{\max }=\left\{x: x \neq x * \text { and } \min _{|x-x *|_{\infty}}\left\{M_{x * x}(x * x)=0\right\}\right\}
$$

and the RoA is then defined by

$$
M_{x x *}(x, x *) \leq M_{x_{\max } x *}\left(x_{\max }, x *\right) \equiv c_{M}
$$

where $c_{M}$ is the maximum value of the distance measure (28) for the particular equilibrium $x *$.

Note that the non-symmetry property of the proposed distance measure (28) allows us to calculate the maximum limit via (30), which clearly is different than the one possibly calculated by setting the initial metric (28) equal to zero. The non-symmetry property enables to determine the widest domain. since it is based on the optimal calculation of $x_{\max }$ for the given $H$. Additionally, when $x_{\max }$ around a particular $x *$ cannot be calculated, then RoA is eliminated, simultaneously meaning instability of $x *$. Hence, in practice. the absence of the symmetry property becomes an advantage.

Although this metric selection may be not the best one to provide the largest stability limits, it has the obvious advantage that $H(x)$ is known from the beginning as the basic storage function used to prove AS of the unforced system and it is usually constructed to express the natural energy function of the system under consideration.

\section{Demonstrating Power System Stability Issues via the Proposed ISS-based Nonlinear Analysis}

Power system stability is a challenging issue of highest priority for the electricity grid operation [1]. Nowadays, the high penetration of renewable energy resources (RES), the continuously encouraged competition in energy market, and the increased load demands have a clear impact on the power system stability. This happens due to many different reasons [28], such as the intermittent nature of RES production, the reduction of the spinning inertia, the need of the electricity market participants to increase their profit by selling more energy, and the targets set by the modern fast developing economies. This new power system technical and economic environment leads the electricity grid to operate close to its technical limits while it is simultaneously under the stress of significant and sometimes unpredictable changing power conditions. Therefore, dynamic secure operation and stability studies of power systems under small or large power disturbances are essential. Small signal analysis-based 
methods have been extensively used in traditional studies [29], but as it is evidently imposed by the new structure and operating conditions, more accurate methods which better fit with the nonlinear nature of the system are required [30]. Thus, in the following subsections, fundamental dynamic and stability issues in power systems analysis are studied in the frame of the massive theoretical infrastructure developed in the previous sections. Since, in power system stability, ISS is mentioned as a significant tool in fundamental references (see e.g., [29]), the based on the ISS notion theoretical development as presented in this paper, provides a complete insight to this direction. Hence, it is shown in detail how the proposed advanced ISS-based nonlinear methods can be applied on power system examples. To demonstrate the effectiveness of the proposed methodology, the simple single generator infinite bus (SGIB) system model is firstly analyzed [31]. In the sequel, it is indicated how this model can be used when the dynamics of reheating cycles are inserted, and it therefore provides the way of studying, in future research works, more complex system configurations with, for example, the $q$-axis electromotive force changing [32] or with interconnections to other generators involved [33].

\subsection{Stability Analysis of the Single Generator Infinite Bus (SGIB) Power System}

The single generator infinite bus (SGIB) power system is a fundamental representation of a power plant connected to a main power grid with much greater capacity than that of the particular plant. The model, given by equations (32) and (33), has been widely used in studying and understanding the power system dynamics and stability as a response of the mechanical power input changes and the speed governor mechanism [31]. Also, the response after large electrical power changes, such as faults, has been studied via the same model [34]. Denoting by $\delta$ the rotor angle, $\omega$ the rotor speed, and $\omega_{0}$ the synchronous speed, where $\Delta \omega=\omega-\omega_{0}$, with $H_{i}$ the generator inertia time constant, $D$ the generator damping coefficient, $X_{d \sigma}^{\prime}$ the $d$-axis synchronous transient reactance, $E_{q}^{\prime}, V_{s}$ the generator transient $q$-axis electromotive force and the infinite bus voltage, respectively, the SGIB model is described by the equations:

$$
\begin{gathered}
\dot{\delta}=\Delta \omega \\
\Delta \dot{\omega}=-\frac{D}{2 H_{i}} \Delta \omega-\frac{\omega_{0}}{2 H_{i}} \frac{E_{q}^{\prime} V_{s}}{X_{d \sigma}^{\prime}} \sin \delta+\frac{\omega_{0}}{2 H_{i}} P_{m}
\end{gathered}
$$

where $P_{m}$ is the external mechanical input which obviously is bounded by

$$
P_{m} \leq P_{m, \max }, P_{m, \max }=\frac{E_{q}^{\prime} V_{s}}{X_{d \sigma}^{\prime}}
$$

As the SGIB model is of the general nonlinear form of (1), with state $x=\left[\begin{array}{cc}\delta & \Delta \omega\end{array}\right]^{T}$ satisfying all the initially considered properties in Section 2, it is easy to apply the proposed theoretical results in order to study its stability properties. Indeed, in accordance to the results deployed in Section 3, the unforced system of (32) and (33) is first considered by setting the external input $P_{m}$ equal to zero $\left(P_{m}=0\right)$. Then, simply constructing the Lyapunov storage function to be equal to the total natural energy of the system as

$$
H_{t} \equiv H_{\omega}+H_{\delta}=\frac{1}{2} \Delta \omega^{2}+\frac{\omega_{0}}{2 H_{i}} \frac{E_{q}^{\prime} V_{s}}{X_{d \sigma}^{\prime}}(1-\cos \delta)
$$

where $H_{\omega}(\Delta \omega), H_{\delta}(\delta)$ represent the kinetic and potential energy of the system and are referred to the first and the second terms of (34), respectively.

Calculating the time derivative of (34),

$$
\dot{H}_{t}=-\frac{D}{2 H_{i}} \Delta \omega^{2} \leq 0
$$


it is directly concluded by applying the LaSalle local invariant set Theorem that the origin of the unforced nonlinear system (32) and (33) is AS.

Then, Corollary 1 guarantees that the complete enforced system states converge to their equilibria as these are calculated by putting the derivatives of $\delta, \Delta \omega$ in (32) and (33) equal to zero, i.e.,

$$
x *=\left[\begin{array}{c}
\delta * \\
\Delta \omega *
\end{array}\right], \text { where } \delta *=\sin ^{-1}\left(\frac{P_{m}}{P_{m, \max }}\right), \Delta \omega *=0
$$

In the domain $-\pi<\delta<\pi$, one stable and another unstable (as shown in the next) equilibria are determined. Therefore, for the system states, it holds true that they locally asymptotically converge to the stable equilibrium.

However, from Corollary 1, it is not easy to obtain the bound given by (7), the proposed method in Section 4 of determining the RoA is applied and described in detail.

To proceed with the RoA, we formulate the following distance measure around the equilibrium using (28), where it is now: $H(x)=H_{t}(x)$.

$$
\begin{aligned}
M_{x x *}(x, x *) & =H_{t}(x)-H_{t}(x *)-\left\langle\nabla H_{t}(x *), x-x *\right\rangle \\
& =H_{\delta}(\delta)-H_{\delta}(\delta *)-\frac{\partial H_{\delta}(\delta *)}{\partial \delta}(\delta-\delta *)+H_{\omega}(\Delta \omega)-\frac{\partial H_{\omega}(0)}{\partial \Delta \omega} \Delta \omega
\end{aligned}
$$

The last two terms in (37) are summarized to $H_{\omega}=\frac{1}{2} \Delta \omega^{2}$ whereas after some manipulations to the three first terms, (37) becomes

$$
M_{x x *}(x, x *)=\frac{\omega_{0}}{2 H_{i}} \frac{E_{q}^{\prime} V_{s}}{X_{d \sigma}^{\prime}}[(\cos \delta *-\cos \delta)-(\delta-\delta *) \sin \delta *]+\frac{1}{2} \Delta \omega^{2}
$$

Since $\dot{M}_{x x *} \leq 0$ and the last term in (38) is always a convex function, the convexity limits of $M_{x x *}$ are dependent from the first term. Therefore, the RoA is evaluated by the symmetric of (38), whereas the last term is cancelled. As determined by (30), condition $M_{x * x}=0$ actually provides the maximum $\delta_{\max }$ and results in the solution of the equation:

$$
\cos \delta_{\max }-\cos \delta *-\left(\delta *-\delta_{\max }\right) \sin \delta_{\max }=0
$$

Therefore, for any bounded $P_{m}$ which approaches a constant value $\bar{P}_{m}>0$ as $t \rightarrow \infty$, a particular equilibrium is obtained from (36) in the range $0<\delta *<\pi / 2$ and another one at $\pi-\delta *$. As clarified in the next paragraphs, for the first equilibrium, a unique value of the $\delta_{\max }$ is calculated by solving (39). Then, the RoA is determined from (31) with

$$
\begin{aligned}
c_{M} & =\frac{\omega_{0}}{2 H_{i}} \frac{E_{q}^{\prime} V_{s}}{X_{s}^{\prime}}\left[\left(\cos \delta *-\cos \delta_{\max }\right)-\left(\delta_{\max }-\delta *\right) \sin \delta *\right] \\
& =\frac{\omega_{0}}{2 H_{i}} \frac{V_{q}^{\prime} V_{s}}{X_{d \sigma}^{\prime}}\left(1+\frac{\sin \delta *}{\sin \delta_{\max }}\right)\left(\cos \delta *-\cos \delta_{\max }\right)
\end{aligned}
$$

where the second expression is obtained by taking into account (39).

In order to evaluate the system performance in accordance with the developed theoretical infrastructure, a power system with parameters given in Table 1 is considered (where pu stands for "per unit").

Before proceeding with the system analysis, Figure 1 is used to clarify some main points with respect to the proposed distance measure and the RoA evaluation. This measure, as given by (38), is dependent from both the system states. However, since the second term of (38) is quadratic and therefore convex, we focus on the first term, which, in Figure 1, represents the vertical distance of every point $\delta$ of function $H_{\delta}(\delta)$ from Line 1 (tangent to $H_{\delta}(\delta)$ at equilibrium $\delta *$ ) as long as this distance is positive. 
Table 1. Parameters of the systems under consideration.

\begin{tabular}{ccc}
\hline Parameter & Value & Units \\
\hline$\omega_{0}$ & $2 \pi 50$ & $\mathrm{rad} / \mathrm{s}$ \\
$E_{q}^{\prime}$ & 1.05 & $\mathrm{pu}$ \\
$V_{s}$ & 1 & $\mathrm{pu}$ \\
$D$ & 5 & $\mathrm{pu}$ \\
$H_{i}$ & 3 & $\mathrm{~s}$ \\
$X_{d \sigma}^{\prime}$ & 0.2 & $\mathrm{pu}$ \\
$c_{m l}$ & 0.7 & - \\
$c_{h}$ & 0.3 & - \\
$T_{h \sigma}$ & 7 & $\mathrm{~s}$ \\
\hline
\end{tabular}

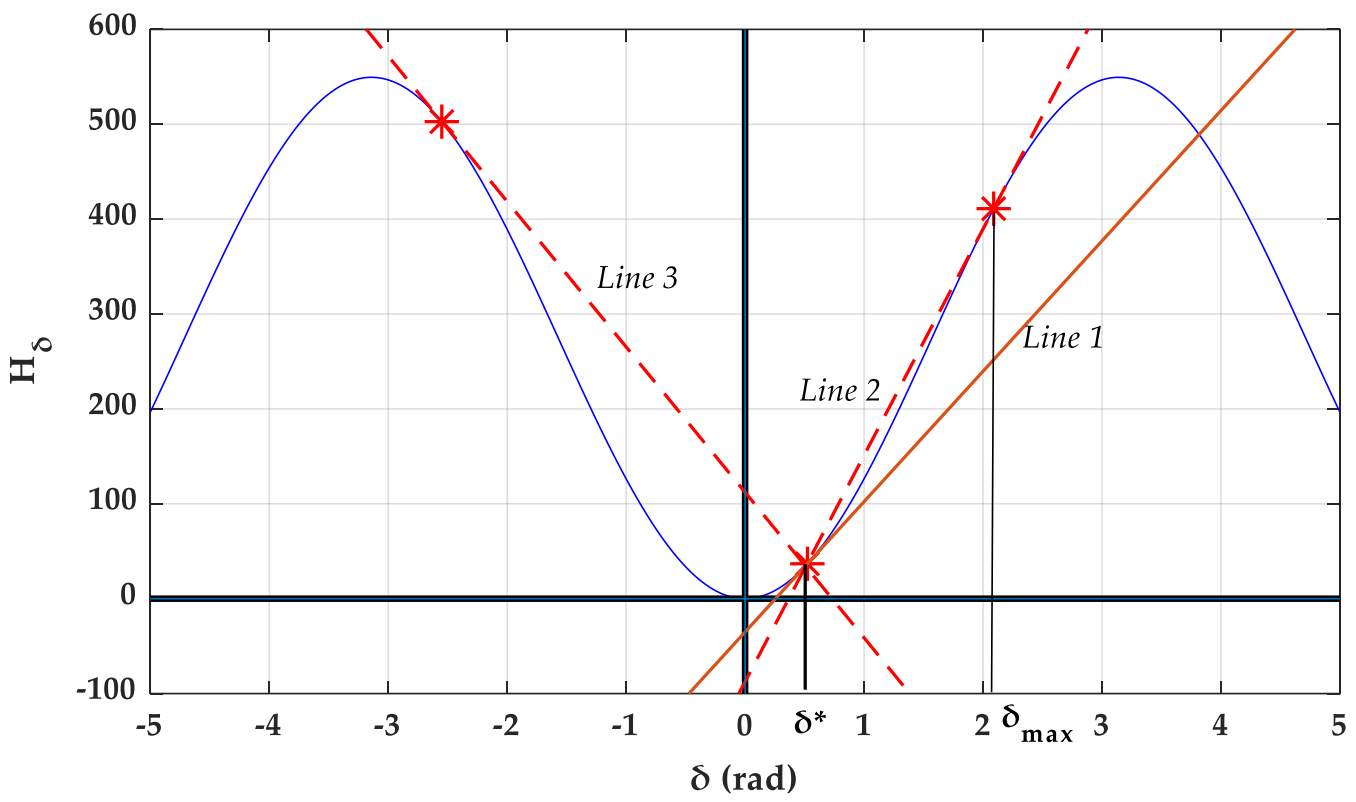

Figure 1. The geometry of $H_{\delta}(\delta)$ explaining the distance measure application on RoA evaluation.

On the other hand, in order to determine $\delta_{\max }$, the symmetric of the aforementioned measure of the $\delta$-part is set equal to zero as defined in (30). This is illustrated by Line 2 , which passes from $\delta *$ and is tangent to function $H_{\delta}(\delta)$. Line 2 clearly determines the range of $\delta$ where $H_{\delta}(\delta)$ is convex as is viewed from the closest to $\delta *$ maximum $\delta_{\max }$ (also, one can see in Figure 1 that Line 3 have the same properties as Line 2, which however determines a wider range and therefore it is not taken into account since violates $\left.\min _{|\delta-\delta *|_{\infty}}\left\{M_{\delta * \delta}=0\right\}\right)$. It is noticed that the particular scheme is taken for $\delta *=\pi / 6$ and the maximum is calculated via (39): $\delta_{\max } \simeq 2 \pi / 3$.

Table 2 provides the maximum $\delta_{\max }$ and the bound $c_{M}$ for different equilibria $\delta *$. One can see that as the equilibrium $\delta *$ increases from 0 to $\pi / 2$, the maximum limit $\delta_{\max }$ decreases. Meanwhile, at the marginal case of $\delta *=\pi / 2$, it is calculated $\delta_{\max }=\pi / 2$, meaning that the RoA, as expected, is eliminated. 
Table 2. The calculated constants $\delta_{\max }$ and $c_{M}$ for different $\delta *$.

\begin{tabular}{ccc}
\hline$\delta^{*}$ & $\delta_{\max }$ & $c_{M}$ \\
\hline 0 & $1.48 \pi / 2$ & 463.87 \\
$\pi / 12$ & $1.41 \pi / 2$ & 290.75 \\
$\pi / 6$ & $1.33 \pi / 2$ & 159.10 \\
$\pi / 4$ & $1.25 \pi / 2$ & 70.31 \\
$\pi / 3$ & $1.17 \pi / 2$ & 21.52 \\
$5 \pi / 12$ & $1.08 \pi / 2$ & 2.70 \\
$\pi / 2$ & $\pi / 2$ & 0 \\
\hline
\end{tabular}

Thus, for any possible equilibrium $\pi / 2 \leq \delta * \leq \pi$, instability is verified since RoA cannot be defined, a fact that distinguishes the stable local equilibrium from the unstable one. It is also remarkable that the $\delta_{\max }=\pi / 2$, which, in practice, is the static upper limit of $\delta$, is very conservative as a dynamic limit, since for all other angles between 0 to $\pi / 2$, the maximum angle is $\delta_{\max }>\pi / 2$. Additionally, it can be easily verified that the RoA, as calculated by the proposed metric, results in a range for the angle close to the one calculated by the equal area criterion [31,35].

Figure 2 shows the RoA for system (32) and (33) for three different equilibria: $\delta *=\pi / 6, \delta *=\pi / 4$, and $\delta *=\pi / 3$. As expected, the RoA decreases as $\delta *$ increases.

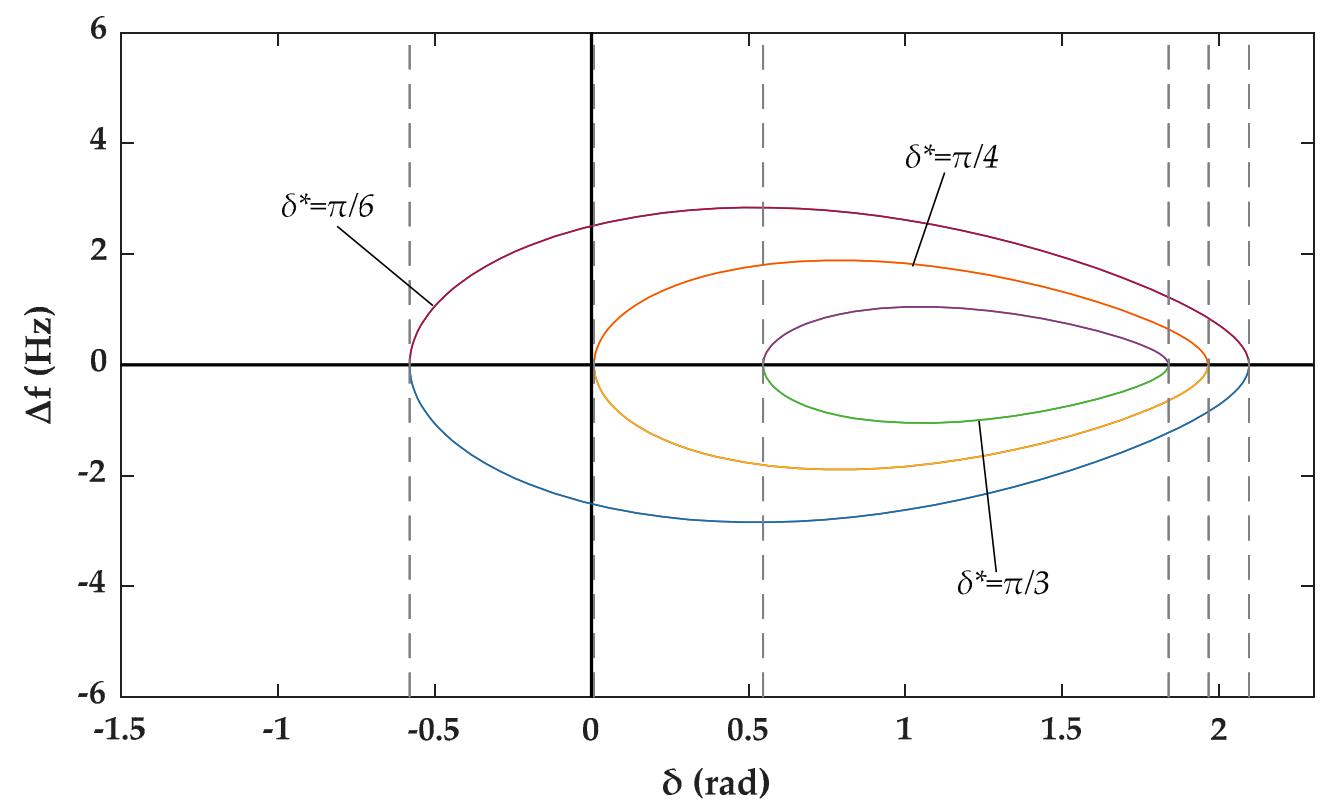

Figure 2. RoA of the single generator infinite bus (SGIB) power system for $\delta *=\pi / 6, \delta *=\pi / 4$, and $\delta *=\pi / 3$.

The response of the system is given in Figures 3 and 4 for angle equilibrium $\delta *=\pi / 6$, as resulted by applying a power input $P_{m}=0.5 P_{m, \max }$. The selected initial conditions, $\delta_{o}=0, \Delta f_{0}=2.54 \mathrm{~Hz}$ (corresponding to $\Delta \omega_{0}=16 \mathrm{rad} / \mathrm{s}$ ), are marginally inside the RoA of Figure 2 in order to examine a difficult case. For the aforementioned stable initial conditions, the convergence of the system states at the stable equilibrium (36) is fully verified. 


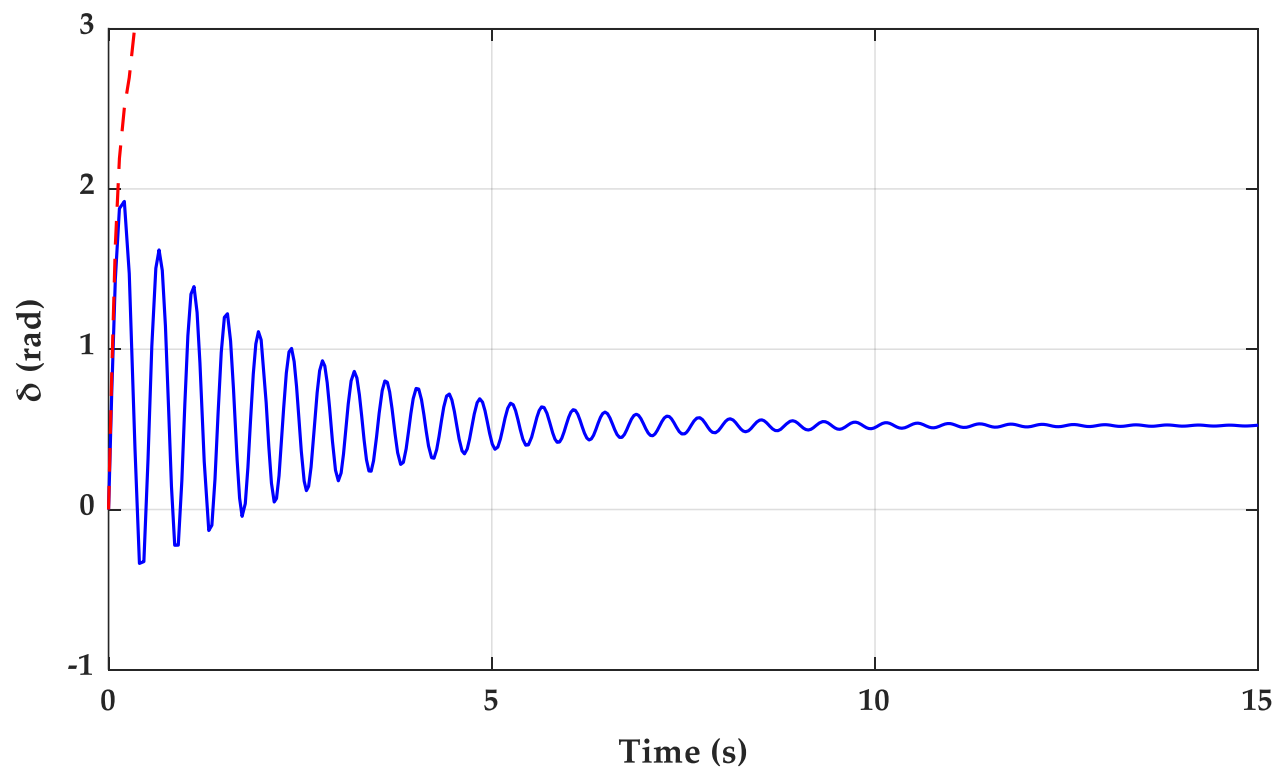

Figure 3. Angle response of the SGIB power system.

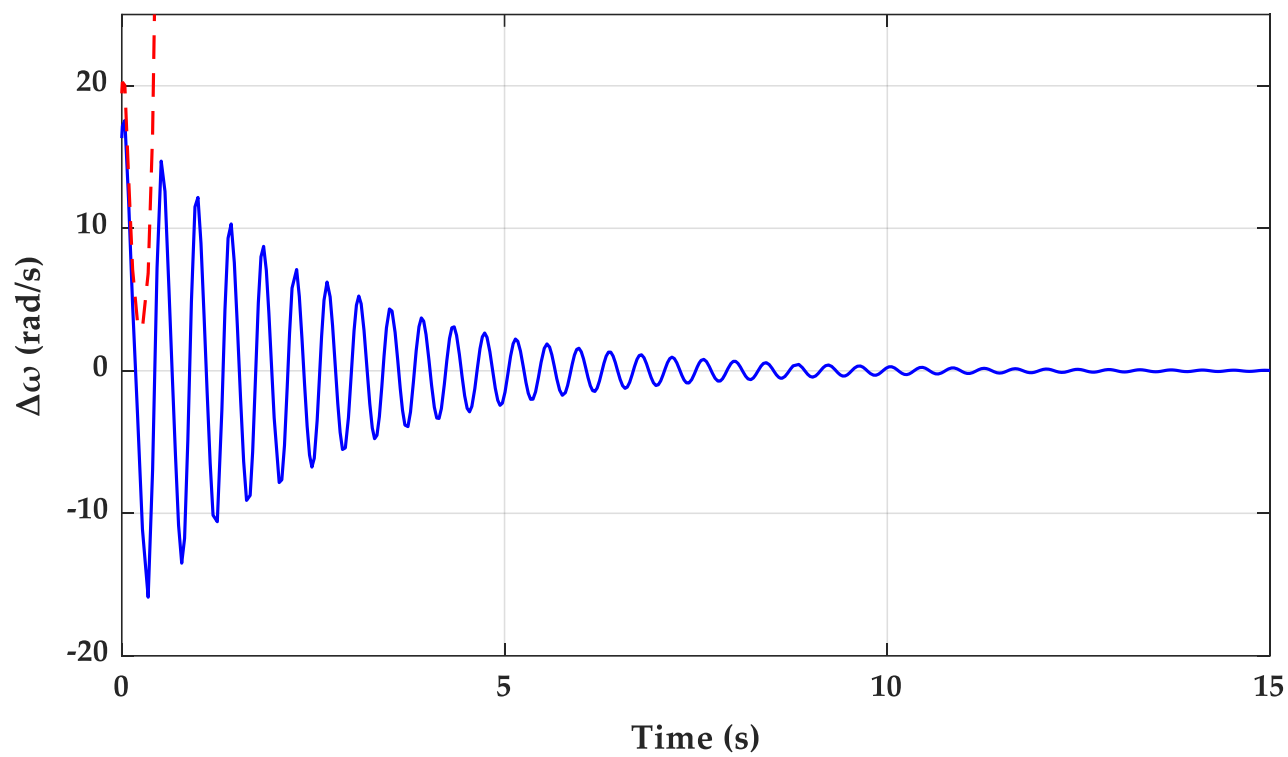

Figure 4. Response of the angular speed of the SGIB power system.

To validate the good evaluation of the RoA, the dotted lines in Figures 3 and 4 show the unstable response of the system for initial values lying marginally outside of the RoA in a place very close to its closure at $\delta_{0}=0, \Delta f_{0}=3.1 \mathrm{~Hz}$ (corresponding to $\Delta \omega_{0}=19.5 \mathrm{rad} / \mathrm{s}$ ). As indicated in Figure 2 and confirmed by the simulations, the stability margins of $\Delta f$ are much more smaller than the angle $\delta$ margins around of acceptable angle equilibriums $\delta *$.

\subsection{Extending SGIB Power System by Considering Reheating Dynamics}

In large-capacity turbine/generator systems, the need of increasing the efficiency of the Rankine thermodynamic cycle requires to use multistage turbines with a high-pressure turbine part, intermediate reheating, and a medium/low-pressure turbine part. Hence, the SGIB power system model that involves an intermediate reheater and two-stage input power dynamics $[31,34]$ becomes:

$$
\dot{\delta}=\Delta \omega
$$




$$
\begin{gathered}
\Delta \dot{\omega}=-\frac{D}{2 H_{i}} \Delta \omega-\frac{\omega_{0}}{2 H_{i}} \frac{E_{q}^{\prime} V_{s}}{X_{d \sigma}^{\prime}} \sin \delta+\frac{\omega_{0}}{2 H_{i}}\left(P_{h}+c_{m l} P_{m}\right) \\
\dot{P}_{h}=-\frac{1}{T_{h \sigma}} P_{h}+\frac{c_{h}}{T_{h \sigma}} P_{m}+\frac{c_{h}}{T_{h \sigma}} v
\end{gathered}
$$

where $P_{h}$ stands for the mechanical power extracted from the high pressure turbine part, whereas the original mechanical power input $P_{m}$ is now split into $c_{m l} P_{m}$ and $c_{h} P_{m}$ at the medium/low-pressure and at the high-pressure turbine parts, respectively. The power distribution weighting coefficients $c_{m l}$ and $c_{h}$ are related by the expression $c_{m l}+c_{h}=1$, usually with values around $c_{m l}=0.7$ and $c_{h}=0.3$. The control input of the steam valve is denoted by $v$, while $T_{h \sigma}$ represents the total time constant of the high-pressure turbine part.

As can been seen from (43), in steady state, $P_{h}=c_{h} P_{m}$ for $v=0$. Therefore, defining $\Delta P_{h}=$ $P_{h}-c_{h} P_{m}$, for some piecewise constant $P_{m}$, model (41)-(43) takes the form

$$
\begin{gathered}
\dot{\delta}=\Delta \omega \\
\Delta \dot{\omega}=-\frac{D}{2 H_{i}} \Delta \omega-\frac{\omega_{0}}{2 H_{i}} \frac{E_{q}^{\prime} V_{s}}{X_{d \sigma}^{\prime}} \sin \delta+\frac{\omega_{0}}{2 H_{i}} \Delta P_{h}+\frac{\omega_{0}}{2 H_{i}} P_{m} \\
\Delta \dot{P}_{h}=-\frac{1}{T_{h \sigma}} \Delta P_{h}+\frac{c_{h}}{T_{h \sigma}} v
\end{gathered}
$$

System (44)-(46) appears to have many similarities in the first two equations with the one previously discussed in (32) and (33). Obviously, the additional third term in the right-hand side of (45) inserts the influence of state $\Delta P_{h}$ in the swing equation, while (46) is independent from Equations (44) and (45). This formulation is characterized as cascaded type between (44), (45), and (46), whereas it can be directly comparable with (32) and (33). For example, for $v=0$, it results $\Delta P_{h}=0$, and then (44) and (45) are identical to (32) and (33). Therefore, the analysis presented in Section 5.1 also holds true for this case.

Now, to proceed with the analysis of the entire model of (44)-(46), it is initially noted that the state

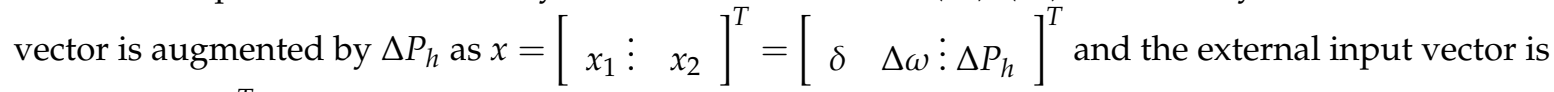
$u=\left[\begin{array}{ll}P_{m} & v\end{array}\right]^{T}$. In accordance to the previous notation, the cascaded form of the system is written as

$$
\begin{aligned}
& \dot{x}_{1}=f_{1}\left(x_{1}, x_{2}, P_{m}\right) \\
& \dot{x}_{2}=f_{2}\left(x_{2}, v\right)
\end{aligned}
$$

with nonzero equilibriua in the domain $-\pi<\delta<\pi$ :

$$
x *=\left[\begin{array}{ll}
x_{1}^{*} & x_{2}^{*}
\end{array}\right]^{T}=\left[\begin{array}{lll}
\delta * & \Delta \omega * & \vdots \Delta P_{h^{*}}
\end{array}\right]^{T}=\left[\begin{array}{lll}
\sin ^{-1}\left(\frac{P_{m}+c_{h} v}{P_{m, m a x}}\right) & 0 & c_{h} v
\end{array}\right]^{T}
$$

where the power input constraint now becomes: $P_{m}+c_{h} v \leq P_{m \text {,max }}$.

Since, for system (47), all the initially considered properties in Section 2 are satisfied, it is again followed the previously used procedure where the unforced system (47) or equivalently (44)-(46) is first considered by setting the external input vector $u$ equal to zero (i.e., $P_{m}=0, v=0$ ).

Then, making use of the cascaded form of the system and recalling from [9] Lemma 4.7, it is sequentially concluded that for the unforced system (47) it holds:

1. The unforced subsystem given by $\dot{x}_{1}=f_{1}\left(x_{1}, x_{2}, 0\right)$ is $l$-ISS by considering $x_{2}$ as external input coming from the cascade subsystem. The proof is exactly the same as in the case of Section 5.1, where now $\Delta P_{h}$ is considered instead of $v$ and, the same Lyapunov storage function $H_{t}$ is used.

2. The unforced cascade subsystem given by $\dot{x}_{2}=f_{2}\left(x_{2}, 0\right)$ is immediately seen to be globally AS since it is a linear time invariant. 
Therefore, in accordance to the aforementioned Lemma 4.7 of [9], the complete unforced system is AS. Applying Corollary 1 for the forced system (44)-(46) with $P_{m} \neq 0, v \neq 0$ and with $P_{m}$ piecewise constant and $v$ approaching a constant value as time passes, it is directly proven that system states asymptotically converge to the equilibrium (48).

The response of the system is taken with initially constant $P_{m}=0.5 P_{m, \max }$ and $v=0$. In the sequel, both of them follow the external input changes as shown in Figure 5, and are presented in Figures 6-8. For comparison reasons, the initial conditions are taken as before: $\delta_{o}=0, \Delta f_{0}=2.54 \mathrm{~Hz}$.

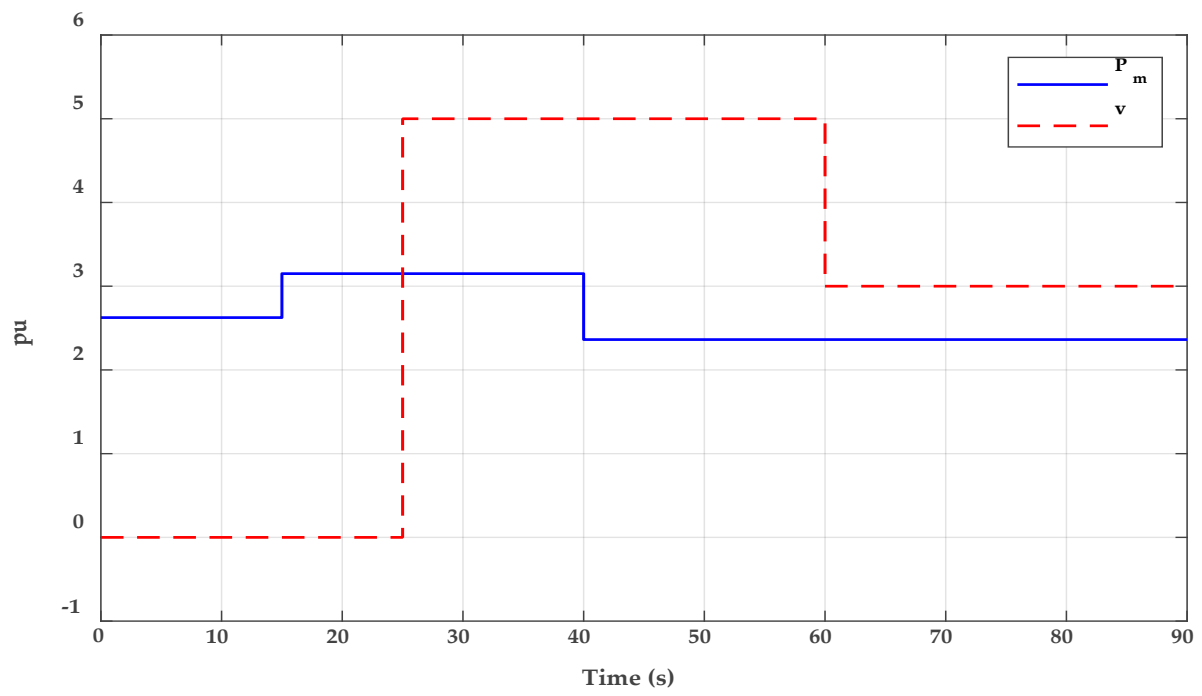

Figure 5. External input profile.

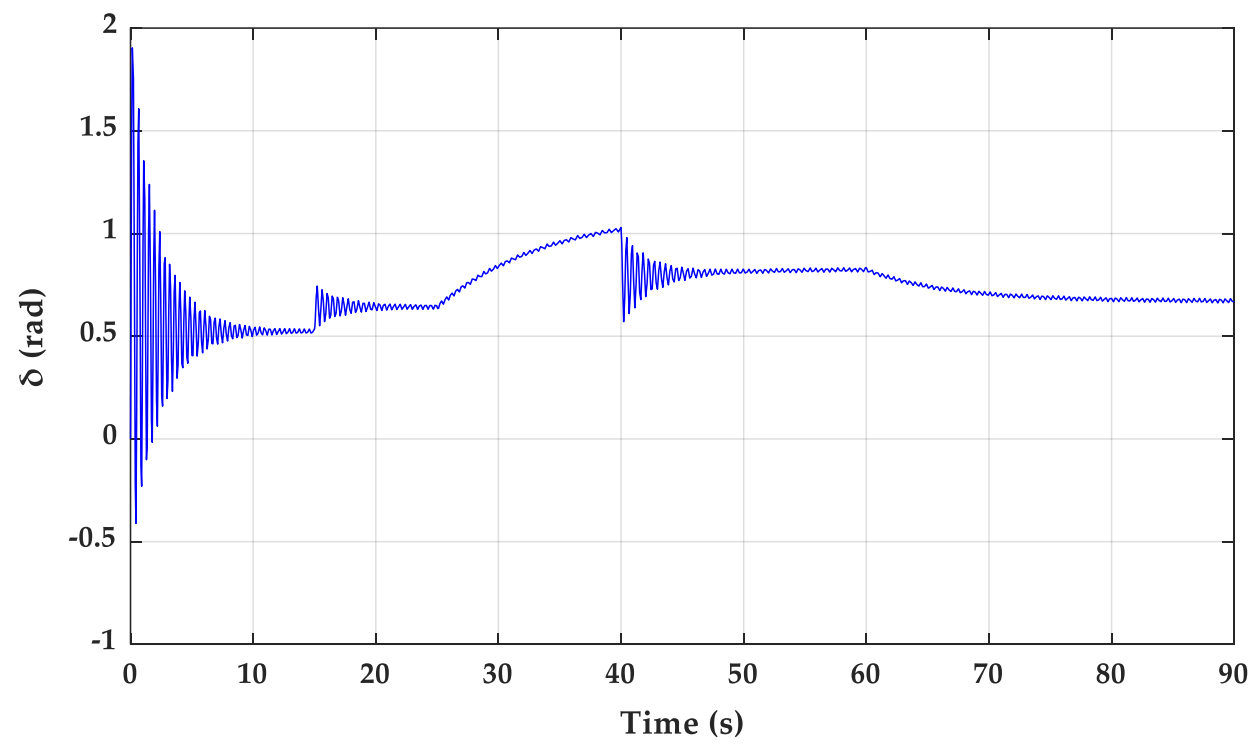

Figure 6. Angle response of the SGIB with reheating.

As expected, the initial system responses are identical to those of the previous case, while when $v$ and $P_{m}$ changed to new constant values, a clear convergence to new steady states occur. As long as the power input constraints, as imposed by (48), are satisfied and the system initial states are inside the RoA, which is initially the same as the discussed in Section 5.1, the system asymptotically converges to the unique stable equilibrium in $-\pi<\delta<\pi$. 


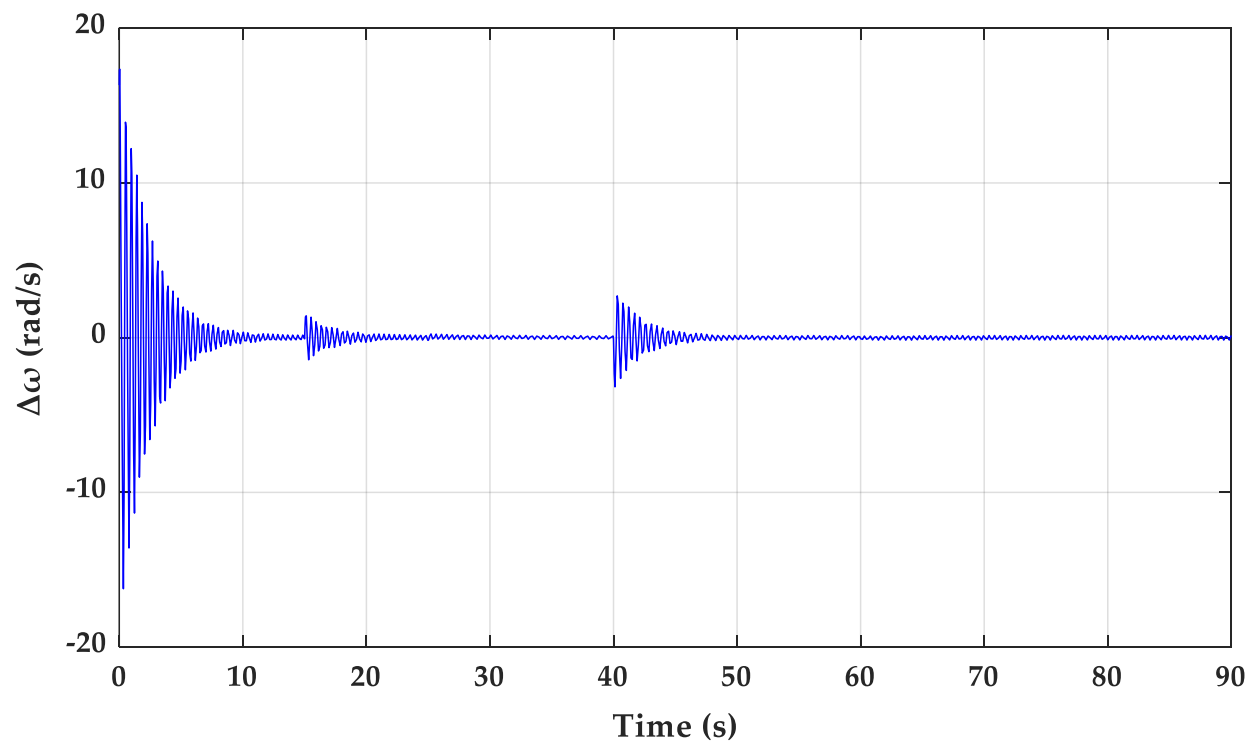

Figure 7. Response of the angular speed deviation of the SGIB with reheating.

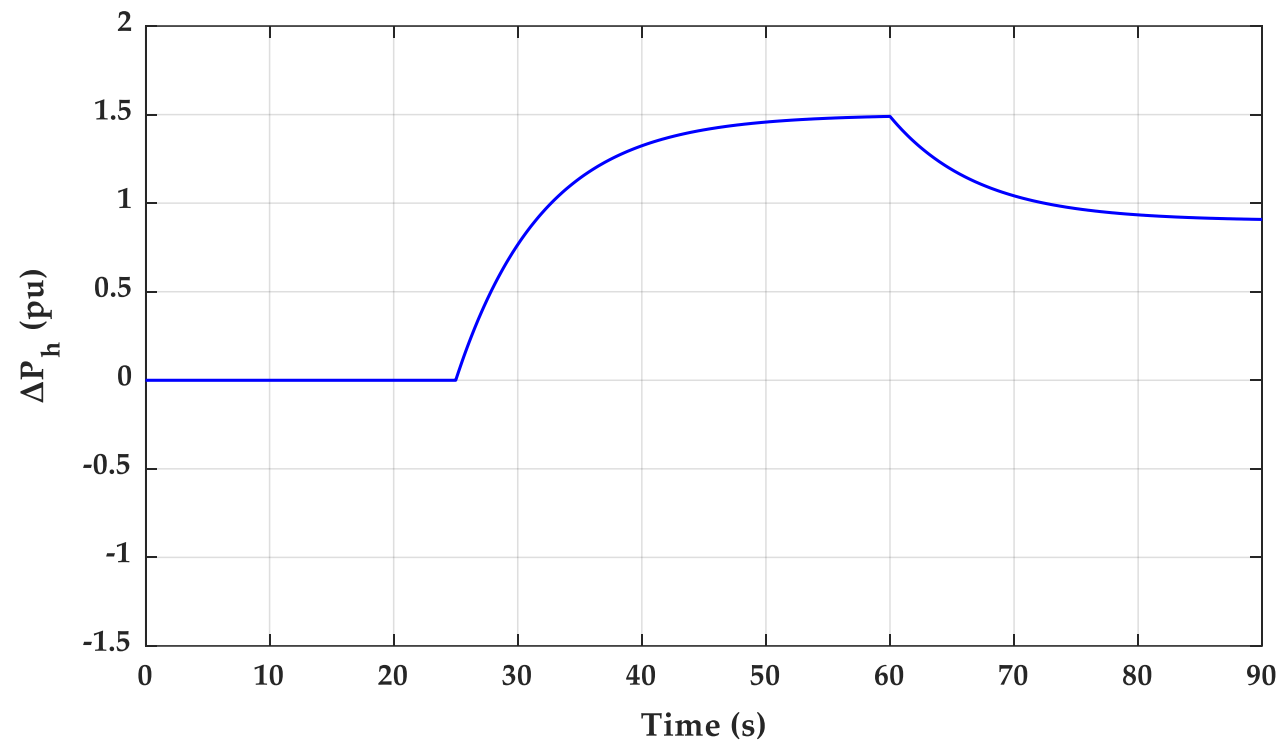

Figure 8. Response of the high-pressure part power deviation of the SGIB with reheating.

\subsection{Inserting Negative $\Delta \omega$-Feedback Control Law}

In this subsection, the proposed method is examined as a tool that enables to us study the different controller impacts acting on the original open-loop system. To illustrate this issue, on the system considered in the last subsection, the well-known $[1,33]$, negative $\Delta \omega$-feedback control law in the steam valve input is generalized into the form

$$
v=-k_{h} \Delta \omega+v_{h}
$$

where $k_{h}>0$, is the arbitrary gain of the controller and $v_{h}$ is the new valve control external input.

Incorporating (49) into (46), the latter equation (46) is substituted by the following equation (50).

$$
\Delta \dot{P}_{h}=-\frac{1}{T_{h \sigma}} \Delta P_{h}-\frac{c_{h}}{T_{h \sigma}} k_{h} \Delta \omega+\frac{c_{h}}{T_{h \sigma}} v_{h}
$$

Now, the system model is given by (44), (45), and (50). 
Examining once again firstly the resulting unforced system by putting $P_{m}=0, v_{h}=0$, it is easier now to prove AS for the controlled externally unforced system, by considering as Lyapunov storage function the total natural energy based function of the system

$$
H_{c} \equiv H_{t}+H_{h} \equiv H_{\omega}+H_{\delta}+H_{h}=\frac{1}{2} \Delta \omega^{2}+\frac{\omega_{0}}{2 H_{i}} \frac{E_{q}^{\prime} V_{s}}{X_{d \sigma}^{\prime}}(1-\cos \delta)+\frac{\omega_{0}}{4 H_{i}} \frac{T_{h \sigma}}{c_{h} k_{h}} \Delta P_{h}^{2}
$$

Then, the time derivative of $H_{c}$ results in

$$
\dot{H}_{c}=-\frac{D}{2 H_{i}} \Delta \omega^{2}-\frac{\omega_{0}}{2 H_{i}} \frac{1}{c_{h} k_{h}} \Delta P_{h}^{2} \leq 0
$$

which can be directly used in the LaSalle local invariant set theorem to complete the proof of AS for the unforced nonlinear system (44), (45), and (50).

Then, Corollary 1 immediately proves that the complete enforced system states asymptotically converge to the new equilibrium

$$
x *=\left[\begin{array}{lll}
\delta * & \Delta \omega * \Delta P_{h}
\end{array}\right]^{T}=\left[\begin{array}{lll}
\sin ^{-1}\left(\frac{P_{m}+c_{h} v_{h}}{P_{m, \max }}\right) & 0 & c_{h} v_{h}
\end{array}\right]^{T}
$$

In Figures 9-11, the system response is presented under the negative $\Delta \omega$-feedback control action implemented with gain value $k_{h}=30$. For comparison reasons, the $P_{m}$ and $v_{h}$ follow the profiles shown in Figure 5. By observing Figure 3 (or Figure 6) and Figure 9, one can clearly see that during the initial transient, the angle response amplitudes are significantly reduced. Certainly, the absorbed energy that enhances the angle response is appeared in the $\Delta P_{h}$ response shown in Figure 11. The same observations can be made by comparing Figures 6-8 with Figures 9-11, respectively, for the cases where $P_{m}$ and $v_{h}$ change their values after the first transient.

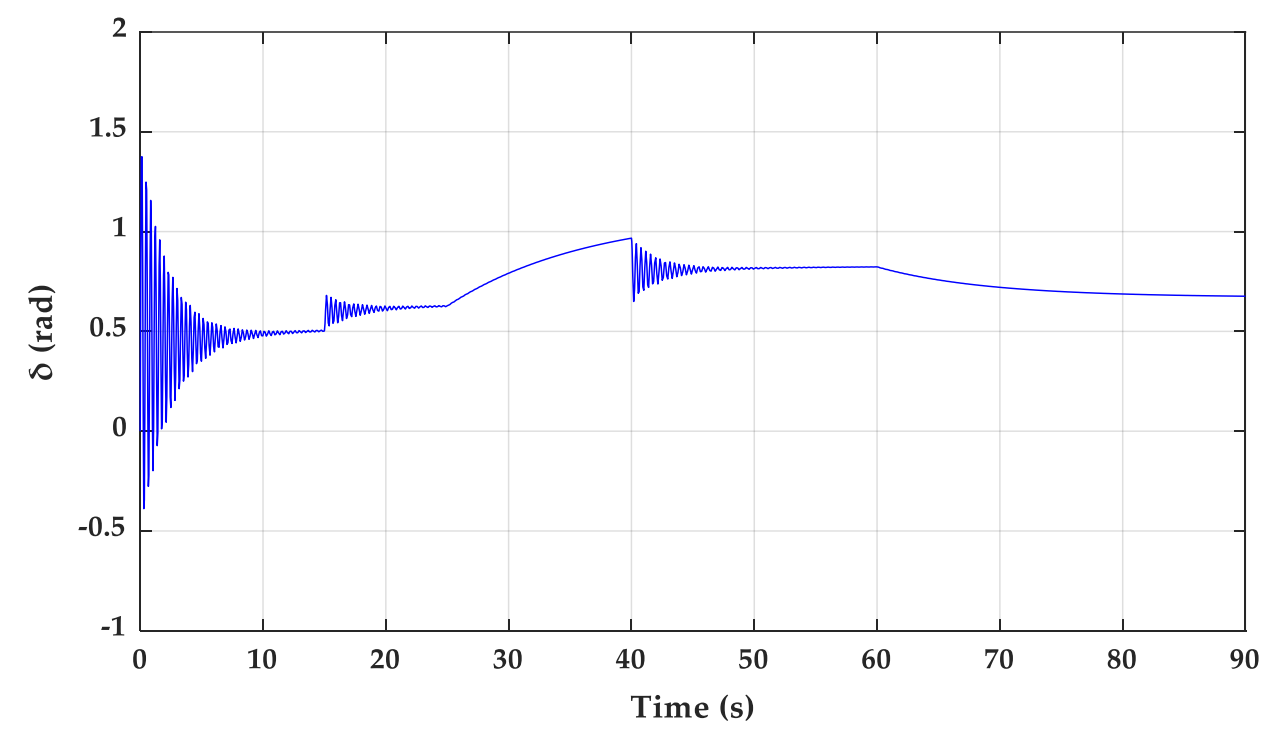

Figure 9. Angle response of the SGIB with feedback.

To further evaluate the influence of the $\Delta \omega$-feedback loop, a case with gain $k_{h}=60$ is considered. As shown in Figure 12, the angle amplitude reduction is greater than the previous one shown in Figure 9, taken with $k_{h}=30$. Therefore, the effectiveness of the inserted $\Delta \omega$-feedback in the high-pressure power loop is confirmed since the angle dynamic performance has been essentially improved. 


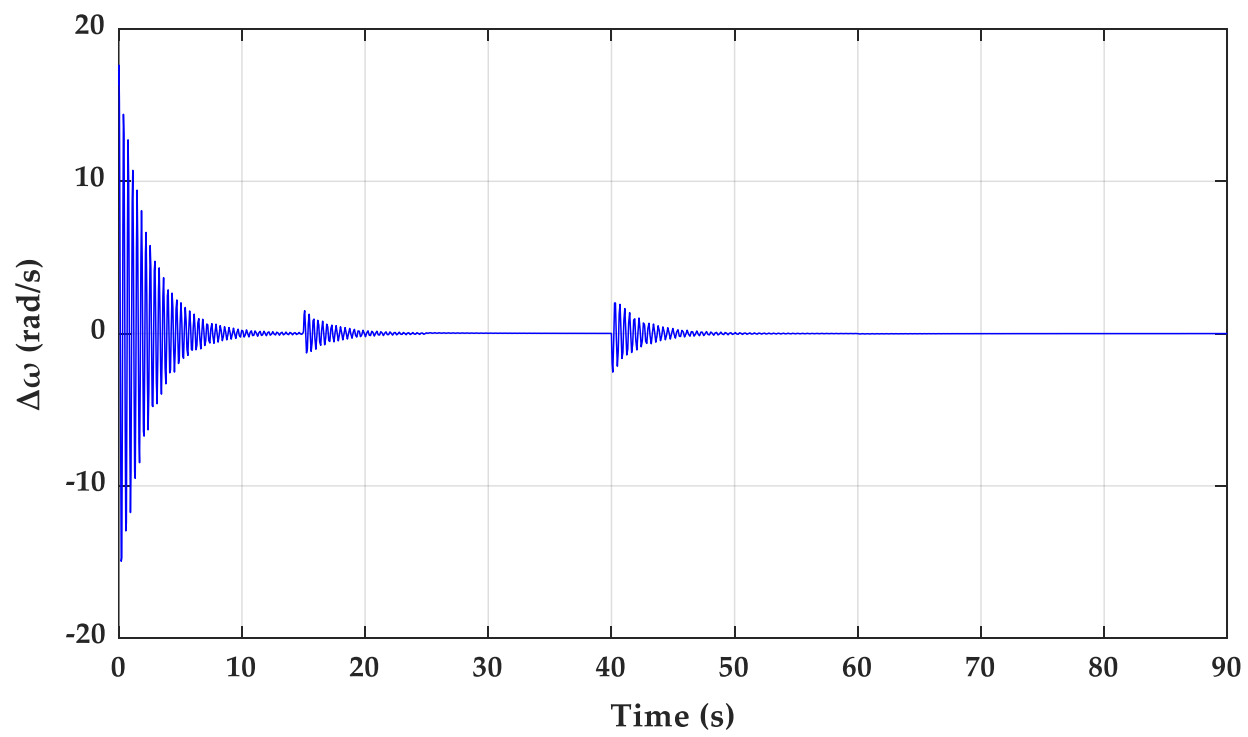

Figure 10. Response of the angular speed of the SGIB with feedback.

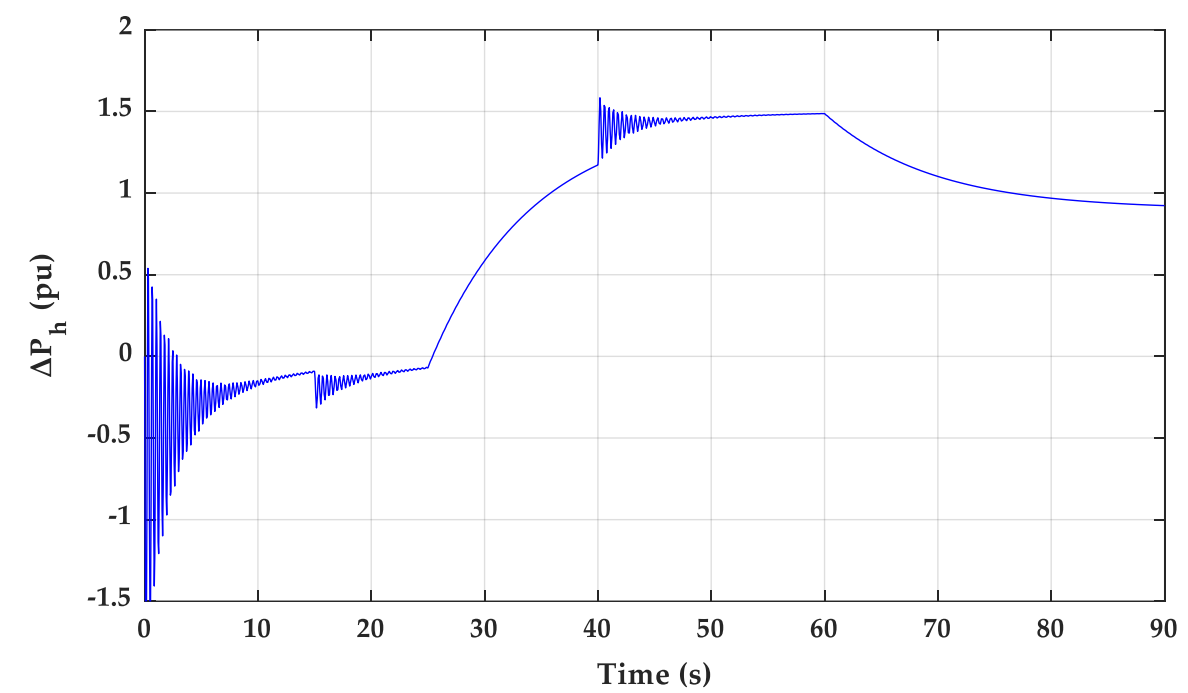

Figure 11. Response of the high-pressure part power deviation of the SGIB with feedback.

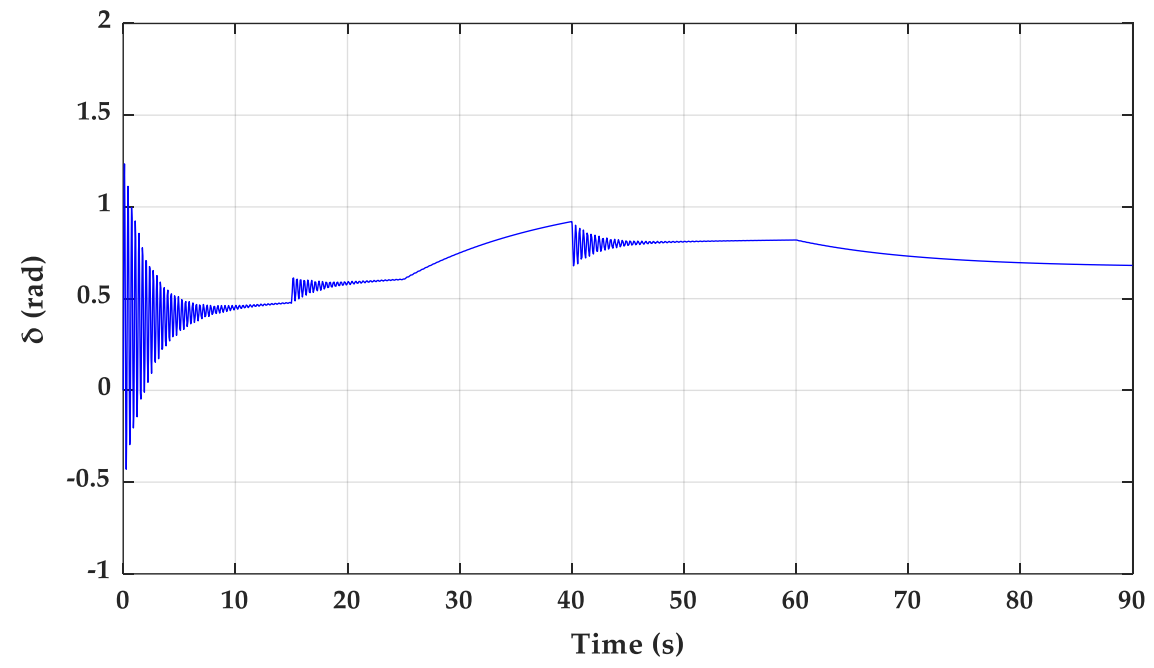

Figure 12. Angle response of the SGIB with high feedback gain. 
It is pointed out that the aforementioned procedure and the integration of the system model of (44)-(46) or (44), (45), and (50) on the basis of the simpler model of (32) and (33) indicate how one can suitably build up more complex power system models in the frame of the proposed theoretical infrastructure. Thus, for example, a varying electromotive force can be considered by inserting its dynamical loop, or even more complex systems can be examined by taking into account interconnected multi-machine schemes [32,36]. Also systems, with power electronic conversion, such as high-voltage direct current (HVDC) transmission systems [37], or with advanced observer based designs [38], can be easily analyzed. Such studies are proposed for future research works, since the present paper mainly focus on the careful and rigorous establishment of a new theoretical analysis approach and the evaluation of the method as an effective tool for nonlinear and practical systems stability applications such the one needed in power systems.

Furthermore, from the previous analysis, it is apparent that the RoA can also be determined in the cases indicated in the last two subsections by following exactly the same procedure as before and therefore, it is not repeated here. It is remarked that for system (44)-(46), when $v \rightarrow 0$ as $t \rightarrow \infty$, the RoA shown in Figure 2 also holds for this case. In nonzero $v$ situations, the RoA is represented in 3D diagrams for different values of $v$. Analogous remarks for the RoA evaluation can be addressed also for systems (44), (45), and (50) where $v_{h}$ now substitutes $v$ as input. In large scale power systems, it is obvious that the RoA is determined only algebraically through (31).

\section{Conclusions}

A novel advanced Lyapunov-based, nonlinear analysis was deployed in this paper, which is suitable for stability studies of externally enforced dynamical systems. The established theorems, and especially the corollaries, provide an effective and easily implemented tool for system analysis with main characteristics: The possibility of considering the natural system energy as a basic Lyapunov storage function and the substantial simplification of the analysis of enforced systems to the well-known analysis of unforced systems. Power systems are characteristic examples of this kind of nonlinear systems where the proposed method is applied. Hence, by conducting a detailed stability analysis in classical nonlinear models of power systems, it was fully validated that the method is quite easy to be applied and to provide more insights into the system behavior, its limits, and its possibility for enhancements through for example suitable control designs. In future works, all of these features are expected to be further exploited and extended to more complex situations.

Funding: No external funding received.

Acknowledgments: The author would like to express his deep grateful to his student Panos Papageorgiou for discussions and his help on extracting and well demonstrating the results in all the figures.

Conflicts of Interest: The author declares no conflicts of interest.

\section{References}

1. Kundur, P. Power System Stability and Control; McGraw-Hill: New York, NY, USA, 1994.

2. Gurrala, G.; Sen, I. Power system stabilizers design for interconnected power systems. IEEE Trans. Power Syst. 2010, 25, 1042-1051. [CrossRef]

3. Sun, Y.Z.; Song, Y.H.; Li, X. Novel energy-based Lyapunov function for controlled power systems. IEEE Power Eng. Rev. 2000, 20, 55-57. [CrossRef]

4. Vu, T.L.; Turitsyn, K. Lyapunov functions family approach to transient stability assessment. IEEE Trans. Power Syst. 2016, 31, 1269-1277. [CrossRef]

5. Ghandhari, M.; Andersson, G.; Hiskens, I.A. Control Lyapunov functions for controllable series devices. IEEE Trans. Power Syst. 2001, 16, 689-694. [CrossRef]

6. Fiaz, S.; Zonetti, D.; Ortega, R.; Scherpen, J.M.A.; van der Schaft, A.J. On port-Hamiltonian modeling of the synchronous generator and ultimate boundedness of its solutions. IFAC Proc. Vol. 2012, 45, 30-35. [CrossRef]

7. Slotine, J.-J.E.; Li, W. Applied Nonlinear Control, 1st ed.; Prentice-Hall: Englewood Cliffs, NJ, USA, 1991. 
8. Banadaki, A.D.; Mohammadi, F.D.; Feliachi, A. State space modeling of inverter based microgrids considering distributed secondary voltage control. In Proceedings of the 2017 North American Power Symposium (NAPS), Morgantown, WV, USA, 17-19 September 2017; pp. 1-6.

9. Khalil, H.K. Nonlinear Systems, 3rd ed; Prentice-Hall: Upper Saddle River, NJ, USA, 2002.

10. Sontag, E.D. Smooth stabilization implies coprime factorization. IEEE Trans. Autom. Control 1989, 34, 435-443. [CrossRef]

11. Marquez, H.J. Nonlinear Control Systems; Wiley \& Sons, Inc.: Hoboken, NJ, USA, 2003.

12. Chaillet, A.; Angeli, D.; Ito, H. Combining iISS and ISS with respect to small inputs: The strong iISS property. IEEE Trans. Autom. Control 2014, 59, 2518-2524. [CrossRef]

13. Qin, B.; Zhang, X.; Ma, J.; Mei, S.; Hill, D.J. Local input to state stability based stability criterion with applications to isolated power systems. IEEE Trans. Power Syst. 2016, 31, 5094-5105. [CrossRef]

14. Sontag, E.D. Remarks on stabilization and input-to-state stability. In Proceedings of the 28th IEEE Conference on Decision and Control (CDC), Tampa, FL, USA, 13-15 December 1989; pp. 1376-1378.

15. Sontag, E.D.; Wang, Y. New characterizations of input-to-state stability. IEEE Trans. Autom. Control 1996, 41, 1283-1294. [CrossRef]

16. Kellett, C.M. A compendium of comparison function results. Math. Control Signals Syst. 2014, 26, 339-374. [CrossRef]

17. Sontag, E.D. Input to state stability: Basic concepts and results. In Nonlinear and Optimal Control Theory. Lecture Notes in Mathematics, 1st ed.; Nistri, P., Stefani, G., Eds.; Springer: Berlin, Germany, 2008; Volume 1932, pp. 163-220.

18. Sontag, E.D.; Wang, Y. On characterizations of the input-to-state stability property. Syst. Control Lett. 1995, 24, 351-359. [CrossRef]

19. Sontag, E.D. A remark on the converging-input converging-state property. IEEE Trans. Autom. Control 2003, 48, 313-314. [CrossRef]

20. Chesi, G. Estimating the domain of attraction via union of continuous families of Lyapunov estimates. Syst. Control Lett. 2007, 56, 326-333. [CrossRef]

21. Bregman, L.M. The relaxation method of finding the common point of convex sets and its application to the solution of problems in convex programming. USSR Comput. Math. Math. Phys. 1967, 7, 200-217. [CrossRef]

22. Liu, C.; Belkin, M. Clustering with Bregman divergences: An asymptotic analysis. In Proceedings of the 30th Conference on Neural Information Processing Systems (NIPS 2016), Barcelona, Spain, 5-10 December 2016.

23. Balestrino, A.; Crisostomi, E.; Grammatico, S.; Caiti, A. A Bregman nonlinear proximal point algorithm for adaptive control. IFAC Proc. Vol. 2011, 44, 4095-4100. [CrossRef]

24. Sontag, E.D. Input-to-State Stability. In Encyclopedia of Systems and Control; Baillieul, J., Samad, T., Eds.; Springer: London, UK, 2015.

25. Sontag, E.D. Comments on integral variants of ISS. Syst. Control Lett. 1998, 34, 93-100. [CrossRef]

26. Konstantopoulos, G.C.; Alexandridis, A.T. Stability and convergence analysis for a class of nonlinear passive systems. In Proceedings of the 50th Conference on Decision and Control and European. Control Conference (CDC-ECC), Orlando, FL, USA, 12-15 December 2011; pp. 1753-1758.

27. Konstantopoulos, G.C.; Alexandridis, A.T. Generalized nonlinear stabilizing controllers for Hamiltonian passive systems with switching devices. IEEE Trans. Control Syst. Technol. 2013, 21, 1479-1488. [CrossRef]

28. Zhang, D.; Yuan, X. Optimization of active current for large-scale wind turbines integrated into weak grids for power system transient stability improvement. Energies 2017, 10, 1108. [CrossRef]

29. Kundur, P.; Paserba, J.; Ajjarapu, V.; Andersson, G.; Bose, A.; Canizares, C.; Hatziargyriou, N.; Hill, D.; Stankovic, A.; Taylor, C.; et al. Definition and classification of power system stability IEEE/CIGRE joint task force on stability terms and definitions. IEEE Trans. Power Syst. 2004, 19, 1387-1401.

30. Qin, B.; Gao, H.; Ma, J.; Li, W.; Zomaya, A.Y. An Input-to-State Stability-based load restoration approach for isolated power systems. Energies 2018, 11, 597. [CrossRef]

31. Mackhowski, J.; Bialek, J.; Bumby, J. Power System Dynamics and Stability; Wiley \& Sons, Ltd.: Chichester, UK, 1997.

32. Psillakis, H.E.; Alexandridis, A.T. Adaptive gain sliding mode control for multimachine power systems. IFAC Proc. Vol. 2005, 38, 61-66. [CrossRef]

33. Wang, Y.; Cheng, D.; Li, C.; Ge, Y. Dissipative Hamiltonian realization and energy-based $\mathrm{L}_{2}$-disturbance attenuation control of multi-machine power systems. IEEE Trans. Autom. Control 2003, 48, 1428-1433. 
34. Ju, X.; Zhao, P.; Sun, H.; Yao, W.; Wen, J. Nonlinear synergetic governor controllers for steam turbine generators to enhance power system stability. Energies 2017, 10, 1092. [CrossRef]

35. Xue, Y.; Van Custem, T.; Ribbens-Pavella, M. Extended equal area criterion justifications, generalizations, applications. IEEE Trans. Power Syst. 1989, 4, 44-52. [CrossRef]

36. Liu, Z.Q.; Yao, W.; Wen, J.Y. Enhancement of power system stability using a novel power system stabilizer with large critical gain. Energies 2017, 10, 449. [CrossRef]

37. Alexandridis, A.T.; Galanos, G.D. Design of a reduced order observer for optimal decentralized control of HVDC systems. IEEE Trans. Power Syst. 1988, 3, 963-969. [CrossRef]

38. Alexandridis, A.T. Design of output feedback controllers and output observers. IEE Proc. Control Theory Appl. 1999, 146, 108-112. [CrossRef]

(C) 2019 by the author. Licensee MDPI, Basel, Switzerland. This article is an open access article distributed under the terms and conditions of the Creative Commons Attribution (CC BY) license (http://creativecommons.org/licenses/by/4.0/). 